\title{
PUSHOUTS OF EXTENSIONS OF GROUPOIDS BY BUNDLES OF ABELIAN GROUPS
}

\author{
Marius Ionescu, Alex Kumjian, Jean N. Renault, Aidan Sims, \\ AND DANA P. Williams \\ (Received 13 July 2021)
}

\begin{abstract}
We analyse extensions $\Sigma$ of groupoids $\mathcal{G}$ by bundles $\mathcal{A}$ of abelian groups. We describe a pushout construction for such extensions, and use it to describe the extension group of a given groupoid $\mathcal{G}$ by a given bundle $\mathcal{A}$. There is a natural action of $\Sigma$ on the dual of $\mathcal{A}$, yielding a corresponding transformation groupoid. The pushout of this transformation groupoid by the natural map from the fibre product of $\mathcal{A}$ with its dual to the Cartesian product of the dual with the circle is a twist over the transformation groupoid resulting from the action of $\mathcal{G}$ on the dual of $\mathcal{A}$. We prove that the full $C^{*}$-algebra of this twist is isomorphic to the full $C^{*}$-algebra of $\Sigma$, and that this isomorphism descends to an isomorphism of reduced algebras. We give a number of examples and applications.
\end{abstract}

We respectfully dedicate this paper to the memory of Vaughan Jones: Extraordinary mathematician, proud New Zealander, and gracious colleague.

\section{Introduction}

There is a significant body of literature regarding the $C^{*}$-algebras of extensions of groupoids by group bundles. The main goal of this paper is to introduce a pushout construction for extensions of groupoids by abelian group bundles and explore its applications.

Specifically, we consider a locally compact Hausdorff groupoid $\mathcal{G}$ together with an abelian group bundle $p_{\mathcal{A}}: \mathcal{A} \rightarrow \mathcal{G}^{(0)}$ where $p_{\mathcal{A}}$ a continuous, open map. Then we consider the following notion of an extension that fixes unit spaces, represented by the diagram

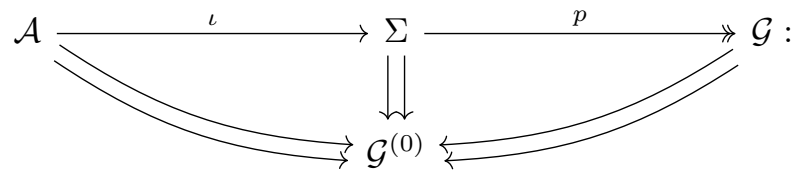

2020 Mathematics Subject Classification 46L05; 20L05.

Key words and phrases: groupoid; $C^{*}$-algebra; pushout.

This research was supported by Australian Research Council grant DP180100595. This work was also partially supported by Simons Foundation Collaboration grants \#209277 (MI), \#353626 (AK) and \#507798 (DPW), and by Grant N0017318WR00251 from the Office of Naval Research and by the Naval Research Laboratory. This work was also facilitated by visits of the second and fifth author to the University of Wollongong as well as a visit by the second author to the Université d'Orléans. We thank our respective hosts and their institutions for their hospitality and support. 
here $\Sigma$ is a locally compact Hausdorff groupoid, $p: \Sigma \rightarrow \mathcal{G}$ is a continuous open surjective groupoid homomorphism that restricts to a homeomorphism $\Sigma^{0} \cong \mathcal{G}^{(0)}$, and $\iota: \mathcal{A} \rightarrow \Sigma$ is a groupooid homomorphism that is a homeomorphism onto $\operatorname{ker}(p)=p^{-1}\left(\mathcal{G}^{(0)}\right)$ in the subspace topology, such that $p \circ \iota=p_{\mathcal{A}}, r_{\Sigma} \circ \iota=r_{\mathcal{A}}=p_{\mathcal{A}}$, $s_{\Sigma} \circ \iota=s_{\mathcal{A}}=p_{\mathcal{A}}, r_{\mathcal{G}} \circ p=r_{\Sigma}$ and $s_{\mathcal{G}} \circ p=s_{\Sigma}$.

A fundamental class of such examples are $\mathbf{T}$-groupoids (also called twists) introduced by the second author in [Kum83]. Then $\mathcal{A}$ is the trivial bundle $\mathcal{G}^{(0)} \times \mathbf{T}$ such that $\iota(r(\sigma), z) \sigma=\sigma \iota(s(\sigma), z)$ for all $\sigma \in \Sigma$ and $z \in \mathbf{T}$. These groupoids and their restricted groupoid $C^{*}$-algebras, $C^{*}(\mathcal{G} ; \Sigma)$, have enjoyed considerable scrutiny [MW92, MW95, Kum83, Kum86]. As usual, in this context we often write $\dot{\sigma}$ in place of $p(\sigma)$.

More recently, we considered more general extensions in [IKSW19] and [IKR $\left.{ }^{+} 21\right]$ as in $(\dagger)$ where it is assumed that $\mathcal{A}$ is endowed with an action of $\mathcal{G}$ and that the extension is compatible in the sense that $\sigma \iota(a) \sigma^{-1}=\iota(\dot{\sigma} \cdot a)$ for all $a \in \mathcal{A}$ and $\sigma \in \Sigma$ such that $p_{\mathcal{A}}(a)=s(\sigma)$.

As a consequence of the main result in $\left[\mathrm{IKR}^{+} 21\right]$, we showed that if $\Sigma$ has a Haar system, then $C^{*}(\Sigma)$ can be realized as the $C^{*}$-algebra of a twist. Specifically, the action of $\mathcal{G}$ on $\mathcal{A}$ induces a natural action of $\mathcal{G}$ on $\hat{\mathcal{A}}$ (regarded as a space). We constructed a $\mathbf{T}$-groupoid $\widetilde{\Sigma}$ of the form

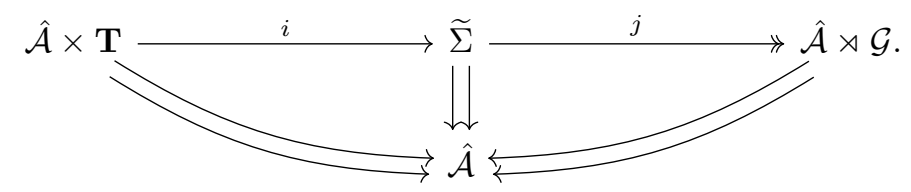

We proved $\left(\left[\mathrm{IKR}^{+} 21\right.\right.$, Theorem 3.4]) that $C^{*}(\Sigma)$ is isomorphic to the restricted $C^{*}$ algebra $C^{*}(\hat{\mathcal{A}} \rtimes \mathcal{G} ; \widetilde{\Sigma})$ of this $\mathbf{T}$-groupoid. (In $\left[\mathrm{IKR}^{+} 21\right]$ the $\mathbf{T}$-groupoid is denoted $\widehat{\Sigma}$, but here we use $\widetilde{\Sigma}$ to avoid possible confusion in our examples.) The T-groupoid $\widetilde{\Sigma}$ is at the heart of the Mackey obstruction which appears in the classical "Mackey machine" of [Mac58].

The chief motivation for this article is the observation that the T-groupoid $\widetilde{\Sigma}$ above - which was based on the construction of [MRW96, Proposition 4.3] - is derived from a natural and functorial "pushout" construction based on the second author's work in [Kum88] for étale groupoids (there called "sheaf groupoids"). Specifically, suppose we are given an extension as in $(\dagger)$, an abelian group bundle $\mathcal{B}$ admitting a $\mathcal{G}$-action, and an equivariant groupoid homomorphism $f: \mathcal{A} \rightarrow \mathcal{B}$. Then there is a similar sort of extension

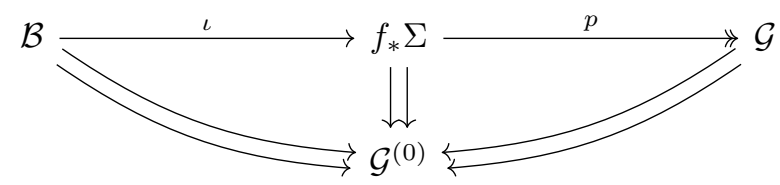

inducing the given $\mathcal{G}$-action on $\mathcal{B}$. In Theorem 1.5 , we show that the construction producing $f_{*} \Sigma$ has good functorial properties that characterize the extension up to a suitable notion of isomorphism. Using these properties, we show in Theorem 2.5 that the collection $T_{\mathcal{G}}(\mathcal{A})$ of isomorphism classes of extensions of $\mathcal{A}$ by $\mathcal{G}$ forms an abelian group (see also [Tu06, §5.3]). 
We close by illustrating how the pushout construction clarifies and interacts with our work in [IKSW19] and $\left[\mathrm{IKR}^{+} 21\right]$. In Theorem 3.2 we prove that the extension $(\ddagger)$ employed in $\left[\mathrm{IKR}^{+} 21\right]$ arises from our pushout construction. Specifically, the natural pairing $(\chi, a) \mapsto \chi(a)$ from $\hat{\mathcal{A}} * \mathcal{A}$ to $\mathbf{T}$ yields a groupoid homomor$\operatorname{phism} f: \hat{\mathcal{A}} * \mathcal{A} \rightarrow \hat{\mathcal{A}} \times \mathbf{T}$ given by $f(\chi, a)=(\chi, \chi(a))$ (see Section 3.1$)$. There is a natural action of $\Sigma$ on $\hat{\mathcal{A}}$ (compatible with that of $\mathcal{G}$ as above) and we prove that $\widetilde{\Sigma} \cong f_{*}(\hat{\mathcal{A}} \rtimes \Sigma)$. This allows us to realise the $C^{*}$-algebra of an extension of a groupoid $\mathcal{G}$ by an abelian group bundle $\mathcal{A}$ as the $C^{*}$-algebra of a $\mathbf{T}$-groupoid over the resulting transformation groupoid $\hat{\mathcal{A}} \rtimes \mathcal{G}$.

Several consequences flow from this observation. First suppose that $\mathrm{A}$ is an abelian group and that $\mathcal{A}=\mathcal{G}^{(0)} \times A$, carrying the action of $\mathcal{G}$ that is trivial in the second coordinate, so that $\Sigma$ is a generalised twist. Each $\chi \in \hat{A}$ defines a homomorphism $f^{\chi}: \mathcal{A} \rightarrow \mathbf{T} \times \mathcal{G}^{(0)}$, so we can form the resulting pushout $f_{*}^{\chi}(\Sigma)$. We prove in Proposition 3.6 that $C^{*}(\Sigma)$ is the section algebra of an upper-semicontinuous $C^{*}$-bundle over $\hat{A}$ with fibres $C^{*}\left(\mathcal{G}, f_{*}^{\chi}(\Sigma)\right)$. When $A$ is compact, this yields a direct sum decomposition which remains valid for the corresponding reduced $C^{*}$ algebras (see Proposition 3.7). In Corollary 3.10 we extend $\left[\operatorname{IKR}^{+} 21\right.$, Theorem 3.4$]$ to the case that $\Omega$ is a $\mathbf{T}$-groupoid extension of $\Sigma$ such that its restriction to $\iota(\mathcal{A})$ is abelian. When $\mathcal{G}$ is étale, this enables us to generalize $\left[\mathrm{IKR}^{+} 21\right.$, Theorem 4.6] to this case (see Corollary 3.11) thereby providing criteria that guarantee that the natural abelian subalgebra of $C_{r}^{*}(\Sigma ; \Omega)$ is Cartan (see also $\left[\mathrm{DGN}^{+} 20\right.$, Theorem 5.8] and [DGN20, Theorem 4.6]).

In Subsection 3.2, we consider the case where the extension $\Sigma$ is determined by an $\mathcal{A}$-valued 2 -cocycle defined on $\mathcal{G}$ and show that the pushout construction is compatible with the natural change of coefficients map on cocycles. We describe the explicit construction of $\widetilde{\Sigma}$ in terms of 2-cocycles at the beginning of Subsection 3.3, and then consider various examples of this construction.

\section{Pushouts of Groupoid Extensions}

We fix a locally compact Hausdorff groupoid $\mathcal{G}$. In our applications, $\mathcal{G}$ will have a Haar system, but this is not required for the pushout construction itself. However, we do assume that $\mathcal{G}$ has open range and source maps. We call a locally compact abelian group bundle $p_{\mathcal{A}}: \mathcal{A} \rightarrow \mathcal{G}^{(0)}$ a $\mathcal{G}$-bundle if $p_{\mathcal{A}}$ is open and $\mathcal{G}$ acts on the left of $\mathcal{A}$ by automorphisms. For compatibility with [IKSW19] - and other examples we have in mind - we will write the group operations in the fibres of such $\mathcal{A}$ additively. An extension $\Sigma$ of $\mathcal{A}$ by $\mathcal{G}$ is determined by a diagram ( $\dagger$ ) as in the introduction. Recall that $\Sigma$ is a locally compact Hausdorff groupoid, $p$ is continuous and open surjection inducing a homeomorphism from $\Sigma^{(0)}$ onto $\mathcal{G}^{(0)}$, and $\iota$ is a continuous open injective homomorphism onto $\operatorname{ker} p=\left\{\sigma \in \Sigma: p(\sigma) \in \mathcal{G}^{(0)}\right\}$. We call such an extension compatible if the action of $\mathcal{G}$ on $\mathcal{A}$ induced by conjugation is the given $\mathcal{G}$-action on $\mathcal{A}$; that is, $\sigma \iota(a) \sigma^{-1}=\iota(\dot{\sigma} \cdot a)$.

Definition 1.1. If $\Sigma_{1}$ and $\Sigma_{2}$ are both compatible extensions by a locally compact abelian group $\mathcal{G}$-bundle $\mathcal{A}$, then we say that they are properly isomorphic if there 
is a groupoid isomorphism $f: \Sigma_{1} \rightarrow \Sigma_{2}$ such that the diagram

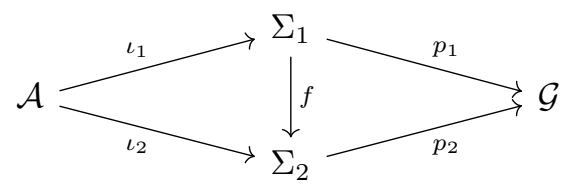

commutes. We let $T_{\mathcal{G}}(\mathcal{A})$ be the collection of proper isomorphism classes of compatible extensions. We denote the equivalence class of a compatible extension $\Sigma$ by $[\Sigma]$.

Remark 1.2. The second author considered extensions of this sort for étale groupoids in $[$ Kum88, §2]. In [Tu06, §5.3], Tu denotes this set by $\operatorname{ext}(\mathcal{G}, \mathcal{A})$ and states that it forms an abelian group (see Theorem 2.5 below). Since the openness of $p_{\mathcal{A}}: \mathcal{A} \rightarrow \mathcal{G}^{(0)}$ implies that $\mathcal{A}$ has a Haar system (see $\left[\mathrm{IKR}^{+} 21\right.$, Lemma 2.1$]$ ), it follows that if $\mathcal{G}$ has a Haar system, then we can then equip $\Sigma$ with a Haar system whenever $[\Sigma] \in T_{\mathcal{G}}(\mathcal{A})\left(\right.$ see $\left[\operatorname{IKR}^{+} 21\right.$, Lemma 2.6]).

Of course, given $\mathcal{G}$ and a $\mathcal{G}$-bundle $\mathcal{A}$, we would like to know that $T_{\mathcal{G}}(\mathcal{A})$ is not empty. To provide a basic example, we follow [Kum88, Definition 2.1].

Example 1.3 (The Semidirect Product). We can build a fundamental compatible extension $\mathcal{A} \triangleleft \mathcal{G}$ from the fibred product $\left\{(a, \gamma) \in \mathcal{A} \times \mathcal{G}: p_{\mathcal{A}}(a)=r(\gamma)\right\}$. We let $(\mathcal{A} \triangleleft \mathcal{G})^{(2)}=\left\{\left(\left(a_{1}, \gamma_{1}\right),\left(a_{2}, \gamma_{2}\right)\right): s\left(\gamma_{1}\right)=r\left(\gamma_{2}\right)\right\}$, and then define

$$
\left(a_{1}, \gamma_{1}\right)\left(a_{2}, \gamma_{2}\right)=\left(a_{1}+\gamma_{1} \cdot a_{2}, \gamma_{1} \gamma_{2}\right) \text { and }(a, \gamma)^{-1}=\left(-\left(\gamma^{-1} \cdot a\right), \gamma^{-1}\right) .
$$

Then we can identify the unit space of $\mathcal{A} \triangleleft \mathcal{G}$ with $\mathcal{G}^{(0)}$ so that $r(a, \gamma)=r(\gamma)$ and $s(a, \gamma)=s(\gamma)$. We can then exhibit $\mathcal{A} \triangleleft \mathcal{G}$ as an extension by letting $\iota(a)=$ $\left(a, p_{\mathcal{A}}(a)\right)$, and letting $p(a, \gamma)=\gamma$. Since

$$
\left(a^{\prime}, \gamma\right)\left(a, p_{\mathcal{A}}(a)\right)\left(-\gamma^{-1} \cdot a^{\prime}, \gamma^{-1}\right)=\left(\gamma \cdot a, p_{\mathcal{A}}(\gamma \cdot a)\right)
$$

$\mathcal{A} \triangleleft \mathcal{G}$ is a compatible extension as required.

Example 1.4. For $i=1,2$ let $\mathcal{A}_{i}$ be a locally compact abelian group $\mathcal{G}$-bundle. Note that $\mathcal{A}_{1} * \mathcal{A}_{2}=\left\{\left(a, a^{\prime}\right): p_{\mathcal{A}_{1}}(a)=p_{\mathcal{A}_{2}}\left(a^{\prime}\right)\right\}$ is also a locally compact abelian group $\mathcal{G}$-bundle. Let $\Sigma_{i}$ be a compatible groupoid extension of $\mathcal{G}$ by $\mathcal{A}_{i}$. Then as in $[$ Kum88, §2], we may form the fibered product

$$
\Sigma_{1} *_{\mathcal{G}} \Sigma_{2}:=\left\{\left(\sigma_{1}, \sigma_{2}\right) \in \Sigma_{1} \times \Sigma_{2} \mid p_{1}\left(\sigma_{1}\right)=p_{2}\left(\sigma_{2}\right)\right\} .
$$

It is straightforward to check that $\Sigma_{1} *_{\mathcal{G}} \Sigma_{2}$ is a compatible groupoid extension of $\mathcal{G}$ by $\mathcal{A}_{1} * \mathcal{A}_{2}$.

Assume now that $\mathcal{B}$ is another abelian group $\mathcal{G}$-bundle, and that $f: \mathcal{A} \rightarrow \mathcal{B}$ is a $\mathcal{G}$-equivariant map. Following [Kum88, Proposition 2.6], we prove that we can "pushout" $\Sigma$ in a unique way to an extension of $\mathcal{G}$ by $\mathcal{B}$.

Theorem 1.5 (Pushout Construction). Let $\mathcal{A}$ and $\mathcal{B}$ be locally compact abelian group $\mathcal{G}$-bundles. Let $f: \mathcal{A} \rightarrow \mathcal{B}$ be a continuous $\mathcal{G}$-equivariant map. Assume that $\Sigma$ is a compatible extension of $\mathcal{G}$ by $\mathcal{A}$. Then there is a compatible extension $f_{*} \Sigma$ 
of $\mathcal{G}$ by $\mathcal{B}$ and a homomorphism $f_{*}: \Sigma \rightarrow f_{*} \Sigma$ such that the following diagram commutes

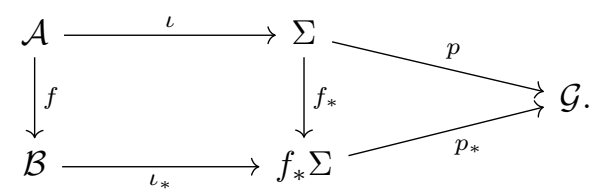

Moreover, $f_{*}$ and $f_{*} \Sigma$ are unique up to proper isomorphism in the sense that if $\Sigma^{\prime}$ is a compatible extension such that the diagram

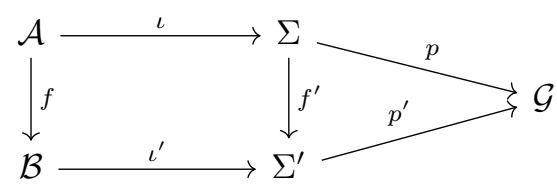

commutes, then there is a proper isomorphism $g: f_{*} \Sigma \rightarrow \Sigma^{\prime}$ such that $g \circ f_{*}=f^{\prime}$.

Proof. Consider the fibred-product groupoid

$$
\mathcal{D}:=(\mathcal{B} \triangleleft \mathcal{G}) *_{\mathcal{G}} \Sigma=\{((b, \gamma), \sigma) \in(\mathcal{B} \triangleleft \mathcal{G}) \times \Sigma: \dot{\sigma}=\gamma\}
$$

of Example 1.4. Define $\theta: \mathcal{A} \rightarrow \mathcal{D}$ via $\theta(a)=\left(\left(-f(a), p_{\mathcal{A}}(a)\right), \iota(a)\right)$. Since $\iota$ is a homeomorphism onto its closed range, $\theta(\mathcal{A})$ is a closed wide subgroupoid of $\mathcal{D}$.

Let $d=((b, \gamma), \sigma) \in \mathcal{D}$. We claim that $d \theta(\mathcal{A})=\theta(\mathcal{A}) d$. To see this, note that

$$
\begin{aligned}
d \theta(a) & =((b, \gamma), \sigma)\left(\left(-f(a), p_{\mathcal{A}}(a)\right), \iota(a)\right) \\
& =((b-\gamma \cdot f(a), \gamma), \sigma \iota(a)) \\
& =\left(\left(-f(\gamma \cdot a)+p_{\mathcal{A}}(\gamma \cdot a) \cdot b, \gamma\right), \iota(\dot{\sigma} \cdot a) \sigma\right) .
\end{aligned}
$$

Since $\dot{\sigma}=\gamma$, we deduce that

$$
\begin{aligned}
d \theta(a) & =\left(\left(-f(\gamma \cdot a), p_{\mathcal{A}}(\gamma \cdot a)\right), \iota(\gamma \cdot a)\right)(b, \gamma, \sigma) \\
& =\theta(\gamma \cdot a) d .
\end{aligned}
$$

Let $f_{*} \Sigma:=\mathcal{D} / \theta(\mathcal{A})$. As usual, we denote the class of $((b, \sigma), \gamma)$ in $f_{*} \Sigma$ by $[(b, \sigma), \gamma]$. Then $[(b, \gamma), \iota(a) \sigma]=[(b+f(a), \gamma), \sigma]$. Since $j(\mathcal{A})$ has a Haar system by Remark 1.2, $f_{*} \Sigma$ is a locally compact Hausdorff groupoid by [IKR ${ }^{+} 21$, Lemma 2.2]. The operations are given by

$$
\begin{aligned}
{\left[\left(b_{1}, \gamma_{1}\right), \sigma_{1}\right]\left[\left(b_{2}, \gamma_{2}\right), \sigma_{2}\right] } & =\left[\left(b_{1}+\gamma_{1} b_{2}, \gamma_{1} \gamma_{2}\right), \sigma_{1} \sigma_{2}\right] \quad \text { and } \\
{[(b, \gamma), \sigma]^{-1} } & =\left[\left(-\gamma^{-1} \cdot b, \gamma^{-1}\right), \sigma^{-1}\right] .
\end{aligned}
$$

We can identify the unit space with $\mathcal{G}^{(0)}$ and then

$$
r([(b, \gamma), \sigma])=r(\gamma) \text { and } s([(b, \gamma), \sigma])=s(\gamma)
$$

To see that $f_{*} \Sigma$ is a compatible extension by $\mathcal{B}$, let

$$
\iota_{*}(b)=\left[\left(b, p_{\mathcal{B}}(b)\right), p_{\mathcal{B}}(b)\right] \quad \text { and } \quad p_{*}([(b, \gamma), \sigma])=\gamma .
$$

It is not hard to verify that this satisfies the algebraic requirements for an extension. The most difficult one might be the inclusion $p_{*}^{-1}\left(\mathcal{G}^{(0)}\right) \subseteq \iota_{*}(\mathcal{B})$ for which we provide an outline of the proof: take $[(b, \gamma), \sigma] \in f_{*} \Sigma$ such that $p_{*}([(b, \gamma), \sigma])=u \in \mathcal{G}^{(0)}$. Then $\gamma=u$, giving $\dot{\sigma}=u$. Since $\Sigma$ is an extension, there exists $a \in \mathcal{A}(u)$ such that $\iota(a)=\sigma$. It follows that $[((b, u), \iota(a))]=[((b+f(a), u), u)]=\iota_{*}(b+f(a))$. It 
is easy to check that $b+f(a)$ is independent of the choice of the representative of $[(b, \gamma), \sigma]$.

Since $\iota_{*}$ and $p_{*}$ are clearly continuous and since $\iota_{*}$ is easily seen to be a homeomorphism onto its range, we just need to see that $p_{*}$ is open. For this, we apply Fell's Criterion (see $\left[\mathrm{IKR}^{+} 21\right.$, Lemma 3.1]). Suppose that $\gamma_{n} \rightarrow \gamma=p_{*}([(b, \sigma), \gamma])$. Since $p: \Sigma \rightarrow \mathcal{G}$ is open, we can pass to a subnet, relabel, and assume that there are $\sigma_{n} \rightarrow \sigma$ in $\Sigma$ such that $\dot{\sigma}_{n}=\gamma_{n}$. Since $p_{\mathcal{B}}$ is open, we can pass to subnet, relabel, and assume there are $b_{n} \rightarrow b$ in $\mathcal{B}$ such that $p_{\mathcal{B}}\left(b_{n}\right)=r\left(\gamma_{n}\right)$. Then $\left[\left(b_{n}, \gamma_{n}\right), \sigma_{n}\right] \rightarrow[(b, \gamma), \sigma]$ as required.

The map $f_{*}$ is the composition of the embedding of $\Sigma$ into $\mathcal{D}$ and the quotient $\operatorname{map} \mathcal{D} \mapsto \mathcal{D} / i(\mathcal{A}): f_{*}(\sigma)=\left[\left(\left(0_{r(\sigma)}, p(\sigma)\right), \sigma\right)\right]$. Since $f$ is $\mathcal{G}$-equivariant, $p_{\mathcal{B}}(f(a))=$ $p_{\mathcal{A}}(a)$ and

$$
f_{*}(\iota(a))=\left[\left(0, p_{\mathcal{A}}(a)\right), p_{\mathcal{A}}(a)\right]=\left[\left(f(a), p_{\mathcal{B}}(f(a))\right), p_{\mathcal{B}}(f(a))\right]=\iota_{*}(\iota(a)),
$$

and $(1.2)$ commutes as required.

Now let $\Sigma^{\prime}$ be an extension as in (1.3). Define $\tilde{g}: \mathcal{D} \rightarrow \Sigma^{\prime}$ by $\tilde{g}((b, \gamma), \sigma)=$ $\iota^{\prime}(b) f^{\prime}(\sigma)$. Since

$$
\iota^{\prime}\left(b_{1}\right) f^{\prime}\left(\sigma_{1}\right) \iota^{\prime}\left(b_{2}\right) f^{\prime}\left(\sigma_{2}\right)=\iota^{\prime}\left(b_{1}\right) \iota^{\prime}\left(f^{\prime}\left(\sigma_{1}\right) \cdot b_{2}\right) f^{\prime}\left(\sigma_{1}\right) f^{\prime}\left(\sigma_{2}\right)
$$

and since $p^{\prime}\left(f^{\prime}\left(\sigma_{1}\right)\right)=\dot{\sigma}_{1}$, it follows that $\tilde{g}$ is a groupoid homomorphism. On the other hand,

$$
\begin{aligned}
\tilde{g}(\theta(a)) & =\tilde{g}\left(\left(-f(a), p_{\mathcal{A}}(a)\right), \iota(a)\right)=\iota^{\prime}(-f(a)) f^{\prime}(\iota(a))=\iota^{\prime}(-f(a)) \iota^{\prime}(f(a)) \\
& =\iota^{\prime}\left(p_{\mathcal{A}}(a)\right) .
\end{aligned}
$$

Hence $\tilde{g}$ factors through a homomorphism $g: f_{*} \Sigma \rightarrow \Sigma^{\prime}$. Clearly, $g\left(\iota_{*}(b)\right)=\iota^{\prime}(b)$ and $p^{\prime} \circ g=p_{*}$, so $g$ makes the diagram analogous to (1.1) commute. We have $g \circ f_{*}=f^{\prime}$ by construction.

To see that $g$ is a proper isomorphism, we still need to see that $g$ is an isomorphism with a continuous inverse.

For this, fix $\alpha \in \Sigma^{\prime}$. There exists $\sigma \in \Sigma$ such that $p(\sigma)=p^{\prime}(\alpha)$. Using (1.3), there exists $b \in \mathcal{B}$ such that $\alpha=\iota^{\prime}(b) f^{\prime}(\sigma)$. So $\tilde{g}$, and hence also $g$, is onto.

Now suppose that $\iota^{\prime}(b) f^{\prime}(\sigma)$ is a unit. Then $f^{\prime}(\sigma)=\iota^{\prime}(-b)$. Hence $p^{\prime}\left(f^{\prime}(\sigma)\right)$ is a unit, and $\sigma=\iota(a)$ for some $a \in \mathcal{A}$. But then $\iota^{\prime}(-b)=f^{\prime}(\sigma)=f^{\prime}(\iota(a))=\iota^{\prime}(f(a))$. Hence, $b=-f(a)$. That is,

$$
((b, p(\sigma)), \sigma)=\left(\left(-f(a), p_{\mathcal{A}}(a)\right), \iota(a)\right) \in \theta(\mathcal{A}) .
$$

Thus $g$ is injective.

To see that $g$ is an isomorphism of topological groupoids, it suffices to see that $g$ is open. We use Fell's criterion. So suppose that $g\left(\alpha_{i}\right) \rightarrow g(\alpha)$ where $\alpha_{i}=$ $\left[\left(b_{i}, p\left(\sigma_{i}\right)\right), \sigma_{i}\right]$ and $\alpha=[(b, p(\sigma)), \sigma] \in f_{*} \Sigma$. Since $p^{\prime} \circ g=p_{*}$, we have $p\left(\sigma_{i}\right) \rightarrow p(\sigma)$. Since $p$ is open, we can pass to a subnet, relabel, and assume there exist $a_{i} \in \mathcal{A}$ such that $\iota\left(a_{i}\right) \sigma_{i} \rightarrow \sigma$. But

$$
\alpha_{i}=\left[\left(-f\left(a_{i}\right)+b_{i}\right), p\left(\sigma_{i}\right), \iota\left(a_{i}\right) \sigma_{i}\right],
$$

and then

It follows that

$$
\iota^{\prime}\left(-f\left(a_{i}\right)+b_{i}\right) f^{\prime}\left(\iota\left(a_{i}\right) \sigma_{i}\right) \rightarrow \iota^{\prime}(b) f^{\prime}(\sigma) .
$$

$$
\iota^{\prime}\left(-f\left(a_{i}\right)+b_{i}\right) \rightarrow \iota^{\prime}(b) .
$$


Since $\iota^{\prime}$ is a homeomorphism onto its range, $\alpha_{i} \rightarrow \alpha$ as required.

Corollary 1.6. Let $\mathcal{A}, \mathcal{B}$ and $\mathcal{C}$ be locally compact abelian group $\mathcal{G}$-bundles. Let $f: \mathcal{A} \rightarrow \mathcal{B}$ and $g: \mathcal{B} \rightarrow \mathcal{C}$ be continuous $\mathcal{G}$-equivariant maps. Assume that $\Sigma$ is a compatible extension of $\mathcal{G}$ by $\mathcal{A}$. Then $(g \circ f)_{*} \Sigma$ is properly isomorphic to $g_{*}\left(f_{*} \Sigma\right)$.

Proof. This follows from the uniqueness of $(g \circ f)_{*} \Sigma$ up to proper isomorphism guaranteed by Theorem 1.5.

\section{The Extension Group $T_{\mathcal{G}}(\mathcal{A})$}

As in $[$ Kum88, §2], we can use our pushout construction to introduce a binary operation on $T_{\mathcal{G}}(\mathcal{A})$. Suppose that $[\Sigma],\left[\Sigma^{\prime}\right] \in T_{\mathcal{G}}(\mathcal{A})$. Define $\nabla^{\mathcal{A}}: \mathcal{A} * \mathcal{A} \rightarrow \mathcal{A}$ by $\nabla^{\mathcal{A}}\left(a, a^{\prime}\right)=a+a^{\prime}$. Proper isomorphisms $f: \Sigma \rightarrow \Gamma$ and $f^{\prime}: \Sigma^{\prime} \rightarrow \Gamma^{\prime}$ of compatible extensions of $\mathcal{A}$ by $\mathcal{G}$ determine a proper isomorphism $f * f^{\prime}: \Sigma * \Sigma^{\prime} \rightarrow \Gamma * \Gamma^{\prime}$ of extensions by $\mathcal{A} * \mathcal{A}$. The uniqueness assertion of Theorem 1.5 then yields a proper isomorphism $\nabla_{*}^{\mathcal{A}}\left(\Sigma *_{\mathcal{G}} \Sigma^{\prime}\right) \rightarrow \nabla_{*}^{\mathcal{A}}\left(\Gamma *_{\mathcal{G}} \Gamma^{\prime}\right)$. Hence the formula

$$
[\Sigma]+\left[\Sigma^{\prime}\right]:=\left[\nabla_{*}^{\mathcal{A}}\left(\Sigma *_{\mathcal{G}} \Sigma^{\prime}\right)\right]
$$

is well defined.

Example 2.1. Let $[\Sigma] \in T_{\mathcal{G}}(\mathcal{A})$. Let $\mathcal{A} \triangleleft \mathcal{G}$ be the semidirect product defined in Example 1.3. Define $g:(\mathcal{A} \triangleleft \mathcal{G}) *_{\mathcal{G}} \Sigma \rightarrow \Sigma$ by $g((a, \dot{\sigma}), \sigma)=\iota(a) \sigma$. We obtain a commutative diagram

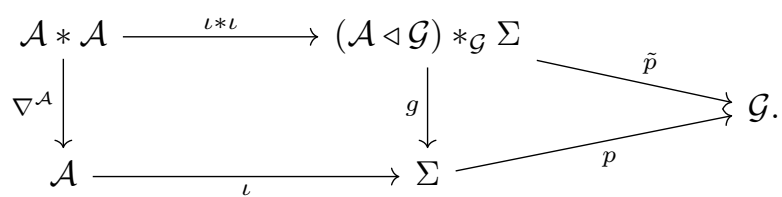

The uniqueness assertion in Theorem 1.5 implies that $\nabla_{*}^{\mathcal{A}}\left((\mathcal{A} \triangleleft \mathcal{G}) *_{\mathcal{G}} \Sigma\right)$ is properly isomorphic to $\Sigma$. In other words, $[\mathcal{A} \triangleleft \mathcal{G}]+[\Sigma]=[\Sigma]$.

Example 2.2. Let $\mathcal{A} \stackrel{\iota}{\longrightarrow} \Sigma \stackrel{p}{\longrightarrow} \mathcal{G}$ be a compatible extension. Then we obtain another compatible extension $\mathcal{A} \stackrel{\iota^{\prime}}{\longrightarrow} \Sigma \stackrel{p}{\longrightarrow} \mathcal{G}$ by letting $\iota^{\prime}(a)=\iota(-a)=\iota(a)^{-1}$. We will write $\Sigma^{-1}$ for $\Sigma$ viewed as this alternate extension. Define $\theta: \mathcal{A} \rightarrow \mathcal{A}$ by $\theta(a)=-a$. Then $\theta$ is $\mathcal{G}$-invariant. Since the diagram

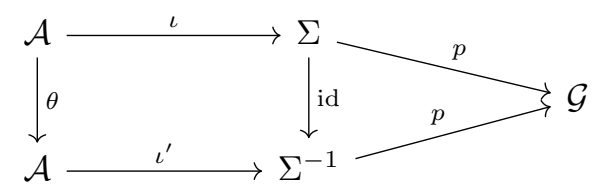

commutes, we can identify $\left[\theta_{*} \Sigma\right]$ with $\left[\Sigma^{-1}\right]$ by Theorem 1.5 .

Example 2.3. Take $[\Sigma] \in T_{\mathcal{G}}(\mathcal{A})$ and let $\mathcal{A} \triangleleft \mathcal{G}$ be the semidirect product. The map $g: \Sigma * \Sigma^{-1} \rightarrow \mathcal{A} \triangleleft \mathcal{G}$ given by $g(\sigma, \tau)=\left(\iota^{-1}\left(\sigma \tau^{-1}\right), \dot{\sigma}\right)$ is a homomorphism. Since 
the diagram

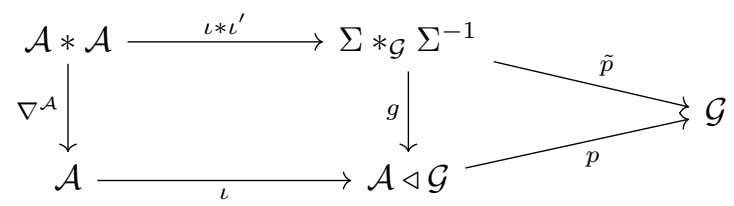

commutes, we see that $[\Sigma]+\left[\Sigma^{-1}\right]=[\mathcal{A} \triangleleft \mathcal{G}]$ for all $\Sigma \in T_{\mathcal{G}}(\mathcal{A})$.

Example 2.4. Take $[\Sigma],\left[\Sigma^{\prime}\right] \in T_{\mathcal{G}}(\mathcal{A})$. Let $\tilde{f}: \Sigma *_{\mathcal{G}} \Sigma^{\prime} \rightarrow \Sigma^{\prime} *_{\mathcal{G}} \Sigma$ be the flip. Similarly, let $f: \mathcal{A} * \mathcal{A} \rightarrow \mathcal{A} * \mathcal{A}$ be given by $f\left(a, a^{\prime}\right)=\left(a^{\prime}, a\right)$. The diagram

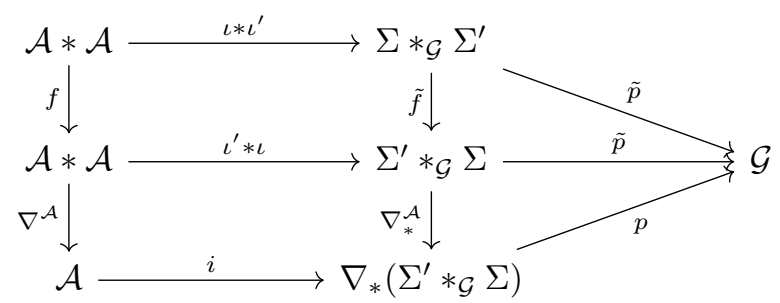

commutes. Since $\nabla^{\mathcal{A}} \circ f=\nabla^{\mathcal{A}}$, it follows from Theorem 1.5 that $[\Sigma]+\left[\Sigma^{\prime}\right]=$ $\left[\Sigma^{\prime}\right]+[\Sigma]$

In Examples 2.1-2.4, we have proved much of the following theorem, which is patterned on [Kum88, Theorem 2.7].

Theorem 2.5. Let $\mathcal{G}$ be a locally compact groupoid with open range and source maps, and let $\mathcal{A}$ be a locally compact abelian group $\mathcal{G}$-bundle. Then the binary operation $\left(\left[\Sigma_{1}\right],\left[\Sigma_{2}\right]\right) \mapsto\left[\nabla_{*}^{\mathcal{A}}\left(\Sigma_{1} *_{\mathcal{G}} \Sigma_{2}\right)\right]$ of $(2.1)$ makes $T_{\mathcal{G}}(\mathcal{A})$ into an abelian group with neutral element given by the class $[\mathcal{A} \triangleleft \mathcal{G}]$ of the semidirect product of Example 1.3, and $[\Sigma]^{-1}=\left[\Sigma^{-1}\right]$ as in Example 2.2. For each continuous $\mathcal{G}$-equivariant map $f: \mathcal{A} \rightarrow \mathcal{B}$ of $\mathcal{G}$-bundles, define $T_{\mathcal{G}}(f): T_{\mathcal{G}}(\mathcal{A}) \rightarrow T_{\mathcal{G}}(\mathcal{B})$ to be the induced map: $T_{\mathcal{G}}(f)[\Sigma]=\left[f_{*} \Sigma\right]$. Then $T_{\mathcal{G}}$ is a functor from the category of $\mathcal{G}$-bundles to the category of abelian groups.

Proof. By considering diagrams similar to that in Example 2.4, we see that the operation in (2.1) is well-defined and associative. We saw that $[\mathcal{A} \triangleleft \mathcal{G}]$ acts as an identity in Example 2.1 and the statement about inverses follows from Example 2.3. The computation in Example 2.4 shows that $T_{\mathcal{G}}(\mathcal{A})$ is an abelian group.

By Corollary 1.6 we have $T_{\mathcal{G}}(f \circ g)=T_{\mathcal{G}}(f) \circ T_{\mathcal{G}}(g)$ if $f$ and $g$ are a composable pair of continuous $\mathcal{G}$-equivariant maps of $\mathcal{G}$-bundles. The proof that $T_{\mathcal{G}}(f)$ is a group homomorphism follows as in the proof of [Kum88, Theorem 2.7].

\section{Applications and Examples}

In this section we consider a unit space fixing extension $\Sigma$ of $\mathcal{G}$ by the group bundle $\mathcal{A}$ as illustrated in the diagram $(\dagger)$ from the introduction. We review the basic details. We assume that all groupoids considered in this section are secondcountable locally compact Hausdorff groupoids with Haar systems. The Haar system on $\Sigma$ is denoted $\lambda=\left\{\lambda^{u}\right\}_{u \in \Sigma^{(0)}}$ and we further assume that $p_{\mathcal{A}}: \mathcal{A} \rightarrow \Sigma^{(0)}$ is a bundle of abelian groups that is a closed subgroupoid of $\Sigma$. It is equipped with a Haar system denoted $\beta=\left\{\beta^{u}\right\}_{u \in \Sigma^{(0)}}$ and the fibers are denoted $\mathcal{A}(u)$ for 
$u \in \Sigma^{(0)}$. The existence of a Haar system on $\mathcal{A}$ implies that $p_{\mathcal{A}}$ is open. It follows by $\left[\mathrm{IKR}^{+} 21\right.$, Lemma $\left.2.6(\mathrm{c})\right]$ that there is a Haar system $\alpha=\left\{\alpha_{u}\right\}_{u \in \Sigma^{(0)}}$ on $\mathcal{G}$ such that for all $f \in C_{c}(\Sigma)$ and $u \in \Sigma^{(0)}$ we have

$$
\int_{\Sigma} f(\sigma) d \lambda^{u}(\sigma)=\int_{\mathcal{G}} \int_{\mathcal{A}} f(\sigma a) d \beta^{s(\sigma)}(a) d \alpha^{u}(\dot{\sigma}) .
$$

Moreover, there is a natural action of $\Sigma$, and therefore $\mathcal{G}$, on $\mathcal{A}$.

Note that $p: \Sigma \rightarrow \mathcal{G}$ is a continuous, open surjection inducing a homeomorphism from $\Sigma^{(0)}$ onto $\mathcal{G}^{(0)}$, and $\iota: \mathcal{A} \rightarrow \Sigma$ is a homeomorphism onto ker $p$. (Both $p$ and $\iota$ are assumed to be groupoid morphisms).

Recall that if $\Sigma$ is a $\mathbf{T}$-groupoid over $\mathcal{G}$ then

$$
C_{c}(\mathcal{G} ; \Sigma):=\left\{f \in C_{c}(\Sigma): f(t \sigma)=t f(\sigma) \text { for all } t \in \mathbf{T}, \sigma \in \Sigma\right\}
$$

is a *-algebra under the operations described in [MW92, §2], and $C^{*}(\mathcal{G} ; \Sigma)$ is its closure in the norm obtained by taking the supremum of the operator norm under all $*$-representations.

We may also view $C_{c}(\mathcal{G} ; \Sigma)$ as compactly supported continuous sections of the one-dimensional Fell line bundle over $\mathcal{G}$ associated to $\Sigma$. One can then construct the associated (right) Hilbert $C_{0}\left(\mathcal{G}^{(0)}\right)$-module $\left(\right.$ see $\left[\mathrm{IKR}^{+} 21, \S 1.3\right]$ ) as the completion of $C_{c}(\mathcal{G} ; \Sigma)$ in the norm arising from the $C_{0}\left(\mathcal{G}^{(0)}\right)$-valued pre-inner product given by $\langle f, g\rangle:=\left.\left(f^{*} * g\right)\right|_{\mathcal{G}^{(0)}}$ for all $f, g \in C_{c}(\mathcal{G} ; \Sigma)$. We denote the Hilbert module by $\mathcal{H}(\mathcal{G} ; \Sigma)$ and observe that left multiplication induces a natural $*$-homomorphism $\lambda: C_{c}(\mathcal{G} ; \Sigma) \rightarrow \mathcal{L}(\mathcal{H}(\mathcal{G} ; \Sigma))$. We may define the reduced norm of an element $f \in C_{c}(\mathcal{G} ; \Sigma)$ to be the operator norm of its image: $\|f\|_{r}:=\|\lambda(f)\|$. Then $C_{r}^{*}(\mathcal{G} ; \Sigma)$ is the closure of $C_{c}(\mathcal{G} ; \Sigma)$ in the reduced norm.

Lemma 3.1. With notation as above, let $F \subset \mathcal{G}^{(0)}$ be a $\mathcal{G}$-invariant clopen subset. Then $F$ is also $\Sigma$-invariant and the reduction $\left.\Sigma\right|_{F}$ is a twist over the reduction $\left.\mathcal{G}\right|_{F}$. Moreover, the characteristic function of $F$ determines a central multiplier projection $p_{F}$ such that

$$
p_{F} C_{r}^{*}(\mathcal{G} ; \Sigma) \cong C_{r}^{*}\left(\left.\mathcal{G}\right|_{F} ;\left.\Sigma\right|_{F}\right) .
$$

Proof. Observe that $\mathcal{H}(\mathcal{G} ; \Sigma)$ decomposes as the direct sum of a Hilbert $C_{0}(F)$ module and a Hilbert $C_{0}\left(F^{c}\right)$-module in the following way

$$
\mathcal{H}(\mathcal{G} ; \Sigma) \cong \mathcal{H}\left(\left.\mathcal{G}\right|_{F} ;\left.\Sigma\right|_{F}\right) \oplus \mathcal{H}\left(\left.\mathcal{G}\right|_{F^{c}} ;\left.\Sigma\right|_{F^{c}}\right) .
$$

Note that multiplication by the characteristic function of $F$, which we denote by $p_{F}$ is the projection onto the first component, that $p_{F}$ is in the center of the multiplier algebra of $C_{r}^{*}(\mathcal{G} ; \Sigma)$, and $C_{c}\left(\left.\mathcal{G}\right|_{F} ;\left.\Sigma\right|_{F}\right)$ acts trivially on the second component. Hence the operator norm of $C_{c}\left(\left.\mathcal{G}\right|_{F} ;\left.\Sigma\right|_{F}\right)$ acting on $\mathcal{H}\left(\left.\mathcal{G}\right|_{F} ;\left.\Sigma\right|_{F}\right)$ coincides with that of its action on $\mathcal{H}(\mathcal{G} ; \Sigma)$.

3.1. The T-groupoid of an extension. As noted in the introduction, we want to see that the $\mathbf{T}$-groupoid constructed in $\left[\mathrm{IKR}^{+} 21, \S 3.1\right]$ is an example of the pushout construction of Theorem 1.5. The $C^{*}$-algebra $C^{*}(\mathcal{A})$ is abelian and the Gelfand dual of $C^{*}(\mathcal{A})$ is an abelian group bundle $\hat{p}: \hat{\mathcal{A}} \rightarrow \mathcal{G}^{(0)}=\Sigma^{(0)}$ with fibres $\hat{p}^{-1}(\{u\}) \cong \mathcal{A}(u)^{\wedge}$ (see [MRW96, Corollary 3.4]). Furthermore, since abelian 
groups are amenable, it follows from [Wil19, Corollary 5.39] and [Wil07, Proposition C.10] that $\hat{p}$ is open. Therefore we can view $\hat{\mathcal{A}}$ as a right $\mathcal{G}$-bundle for the natural right action of $\mathcal{G}$ on $\hat{\mathcal{A}}$.

Since $\mathcal{G}$ and $\Sigma$ both act on $\hat{\mathcal{A}}$, regarded as a topological space fibered over $\Sigma^{(0)}$, we can form the transformation groupoids $\hat{\mathcal{A}} \rtimes \mathcal{G}$ and $\hat{\mathcal{A}} \rtimes \Sigma$. Moreover, $\hat{\mathcal{A}} * \mathcal{A}=\left\{(\chi, a): \hat{p}(\chi)=p_{\mathcal{A}}(a)\right\}$ is a $\hat{\mathcal{A}} \rtimes \mathcal{G}$-bundle (as well as an $\hat{\mathcal{A}} \rtimes \Sigma$-bundle). Defining $\iota_{*}: \hat{\mathcal{A}} * \mathcal{A} \rightarrow \hat{\mathcal{A}} \rtimes \Sigma$ by $\iota_{*}(\chi, a)=(\chi, a)$ and $p_{*}: \hat{\mathcal{A}} \rtimes \Sigma \rightarrow \hat{\mathcal{A}} \rtimes \mathcal{G}$ by $p_{*}(\chi, \sigma)=(\chi, \dot{\sigma})$, we obtain an extension

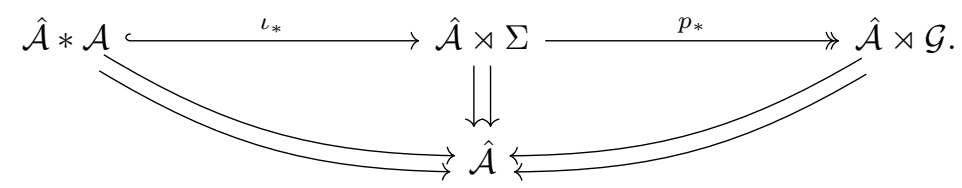

We defined a T-groupoid $\widetilde{\Sigma}$ associated to this extension in [IKR ${ }^{+} 21$, Proposition 3.2] as follows. Define

$$
\mathcal{D}=\{(\chi, z, \sigma) \in \hat{\mathcal{A}} \times \mathbf{T} \times \Sigma: \hat{p}(\chi)=r(\sigma)\}
$$

and let $H$ be the subgroupoid of $\mathcal{D}$ consisting of triples of the form $(\chi, \overline{\chi(a)}, a)$ for $a \in \mathcal{A}(\hat{p}(\chi))$. Then $H$ is a normal subgroupoid of $\mathcal{D}$ and we can form the locally compact Hausdorff groupoid $\widetilde{\Sigma}:=\mathcal{D} / H$ (we use the notation $\widetilde{\Sigma}$, rather than the notation $\widehat{\Sigma}$ of $\left[\mathrm{IKR}^{+} 21\right]$, to avoid clashing with classical notational conventions when $\Sigma$ is a group, for example in Remark 3.3).

Theorem 3.2. Let $\Sigma$ be the extension of $\mathcal{G}$ by the group bundle $\mathcal{A}$ as in the diagram ( $\dagger$ ) and adopt the notation established above. Let $f: \hat{\mathcal{A}} * \mathcal{A} \rightarrow \hat{\mathcal{A}} \times \mathbf{T}$ be the canonical map given by

$$
f(\chi, a)=(\chi, \chi(a)) .
$$

Then $\widetilde{\Sigma}$ is properly isomorphic to the pushout $f_{*}(\hat{\mathcal{A}} \rtimes \Sigma)$. Moreover,

$$
C^{*}(\Sigma) \cong C^{*}\left(\hat{\mathcal{A}} \rtimes \mathcal{G} ; f_{*}(\hat{\mathcal{A}} \rtimes \Sigma)\right) \quad \text { and } \quad C_{r}^{*}(\Sigma) \cong C_{r}^{*}\left(\hat{\mathcal{A}} \rtimes \mathcal{G} ; f_{*}(\hat{\mathcal{A}} \rtimes \Sigma)\right) .
$$

Proof. Theorem 1.5 implies that there is a unique (up to proper isomorphism) extension $f_{*}(\hat{\mathcal{A}} \rtimes \Sigma)$ of $\hat{\mathcal{A}} \rtimes \mathcal{G}$ by $\hat{\mathcal{A}} \times \mathbf{T}$ and a twist morphism that is compatible with $f$. In particular, $f_{*}(\hat{\mathcal{A}} \rtimes \Sigma)$ is a $\mathbf{T}$-groupoid. We get a natural map $g: \hat{\mathcal{A}} \rtimes \Sigma$ to $\widetilde{\Sigma}$ given by $g(\chi, \sigma)=[\chi, 1, \sigma]$, and the diagram

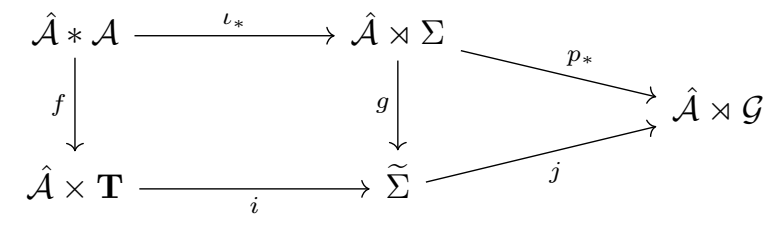

commutes. The proper isomorphism of $\widetilde{\Sigma}$ with $f_{*}(\hat{\mathcal{A}} \rtimes \Sigma)$ follows from the uniqueness guaranteed by Theorem 1.5 and the final assertion follows from $\left[\mathrm{IKR}^{+} 21\right.$, Theorem 3.3].

It follows immediately that if $\Sigma$ is properly isomorphic to the semidirect product $\mathcal{A} \triangleleft \mathcal{G}$, then $[\hat{\mathcal{A}} \rtimes \Sigma]=[\hat{\mathcal{A}} \rtimes(\mathcal{A} \triangleleft \mathcal{G})]=[\mathcal{A} \triangleleft(\hat{\mathcal{A}} \rtimes \mathcal{G})]$ and hence $[\widetilde{\Sigma}]$ is trivial. Thus $C^{*}(\Sigma) \cong C^{*}(\hat{\mathcal{A}} \rtimes \mathcal{G})$. 
Remark 3.3. As mentioned in the introduction, the twist $\widetilde{\Sigma}$ appearing in Theorem 3.2 is responsible for the Mackey obstruction of the classical normal subgroup analysis of [Mac58]. Indeed, let us apply the theorem when $\Sigma$ is a locally compact group and $\mathcal{A}$ is a closed normal abelian subgroup. Then $\Sigma$ and $\mathcal{G}=\Sigma / \mathcal{A}$ act on $\mathcal{A}$ by conjugation and give right actions on the space of characters $\hat{\mathcal{A}}$. The corresponding twist $\widetilde{\Sigma}$ is the quotient of the groupoid $(\hat{\mathcal{A}} \rtimes \Sigma) \times \mathbf{T}$ where $(\chi, a \sigma, \theta)$ is identified with $(\chi, \sigma, \theta \chi(a))$ for all $a \in \mathcal{A}$. We let $[\chi, \sigma, \theta]$ be the class of $(\chi, \sigma, \theta)$ in $\widetilde{\Sigma}$. If $\chi \in \hat{\mathcal{A}}$, then let $\Sigma(\chi)$ and $\mathcal{G}(\chi)$ be the stabilizers at $\chi$ for the actions on $\hat{\mathcal{A}}$, and let $\widetilde{\Sigma}(\chi)$ be the isotropy group of $\widetilde{\Sigma}$ at $\chi$. We observe that $\widetilde{\Sigma}(\chi)$, up to an obvious identification, is the pushout of the group extension

$$
\mathcal{A} \longrightarrow \Sigma(\chi) \longrightarrow \mathcal{G}(\chi)
$$

by the homomorphism $\chi: \mathcal{A} \rightarrow \mathbf{T}$. Indeed, this pushout $\chi_{*}(\Sigma(\chi))$ is the quotient of $\Sigma(\chi) \times \mathbf{T}$ by the equivalence relation identifying $(a \sigma, \theta)$ with $(\sigma, \theta \chi(a))$ for all $a \in \mathcal{A}$. Thus we just identify $[\chi, \sigma, \theta] \in \widetilde{\Sigma}(\chi)$ with $[\sigma, \theta] \in \chi_{*}(\Sigma(\chi))$. The class of $\widetilde{\Sigma}(\chi)$ in $H^{2}(\mathcal{G}(\chi), \mathbf{T})$ is the classical Mackey obstruction. More precisely, let $L$ be an irreducible unitary representation of $\Sigma$. According to Theorem 3.2, we may view it as a representation of the twisted groupoid $(\hat{\mathcal{A}} \rtimes \mathcal{G}, \widetilde{\Sigma})$. Its restriction to $\hat{\mathcal{A}}$ defines a measure class which is invariant and ergodic under the action of $\mathcal{G}$. If this measure class is transitive, which will be always the case if $\mathcal{A}$ is regularly embedded, then we have a representation of a twisted transitive measured groupoid $\left(O \rtimes \mathcal{G},\left.\widetilde{\Sigma}\right|_{O}\right)$, where $O \subset \hat{\mathcal{A}}$ is an orbit of the action and $\left.\widetilde{\Sigma}\right|_{O}$ is the reduction of $\widetilde{\Sigma}$ to $O$. We pick $\chi \in O$. Since the $\left(\widetilde{\Sigma}(\chi),\left.\widetilde{\Sigma}\right|_{O}\right)$-groupoid equivalence $\widetilde{\Sigma}_{O}^{\chi}$ is compatible with the twists in the sense of [Ren87, Définition 5.3], it implements a bijective correspondence between the unitary representations of $\left(O \rtimes \mathcal{G},\left.\widetilde{\Sigma}\right|_{O}\right)$ and those of $(\mathcal{G}(\chi), \widetilde{\Sigma}(\chi))$. Therefore $L$ is given by an irreducible unitary representation of the twisted group $(\mathcal{G}(\chi), \widetilde{\Sigma}(\chi))$.

Example 3.4. Let $H$ be a locally compact abelian group and let $A \subset H$ be a closed subgroup. Then applying the above theorem with $\Sigma=H$ and $\mathcal{A}=A$, we conclude that $\widetilde{\Sigma}$ is a bundle of abelian groups over $\widetilde{\Sigma}^{(0)} \cong \hat{A}$ where each fiber is an extension of $H / A$ by $\mathbf{T}$. Each of these extensions is abelian because $H$ is abelian (and the action of $H$ on $\hat{A}$ is trivial). Hence, each extension is determined by a symmetric $\mathbf{T}$-valued Borel 2-cocycle and any such 2-cocycle is necessarily trivial by [Kle65, Lemma 7.2]. But the twist is not trivial in general: for example, if $H=\mathbf{R}$ and $A=\mathbf{Z} \leq \mathbf{R}$, then triviality of the twist would imply $C^{*}(\mathbf{R}) \cong C_{0}(\mathbf{T} \times \mathbf{Z})$, which is nonsense.

Example 3.5 (Generalized Twists). We now consider the case where $A$ is a locally compact abelian group, $\mathcal{A}=\mathcal{G}^{(0)} \times A$, and $\mathcal{G}$ acts on $\mathcal{A}$ by translation on the first factor. Since this simply gives us a twist when $A=\mathbf{T}$, we will say that $\Sigma$ is a generalized twist in this case. Note that even for twists, $\Sigma$ need not be a trivial extension. Generalized twists were studied in [IKSW19].

View $\hat{\mathcal{A}}:=\hat{A} \times \mathcal{G}^{(0)}$ as a locally compact space. (We put the factor of $\mathcal{G}^{(0)}$ on the right, as a reminder that we are thinking of $\hat{A}$ as a space rather than as a group, and to line up with the natural identification of $\hat{\mathcal{A}} * \mathcal{A}$ with $\hat{A} \times \mathcal{G}^{(0)} \times A$, which we make without further comment). Then $\mathcal{G}$ acts on the second factor of $\hat{\mathcal{A}}$. This means we 
can replace $\hat{\mathcal{A}} \rtimes \mathcal{G}$ and $\hat{\mathcal{A}} \rtimes \Sigma$ with the products $\hat{A} \times \mathcal{G}$ and $\hat{A} \times \Sigma$, respectively. Under these identifications, Equation (3.2) becomes $f(\chi, u, a)=(\chi, u, \chi(a))$. Moreover we may assume that the Haar system $\beta$ on $\mathcal{A}=\mathcal{G}^{(0)} \times A$ is constant in the sense that there is a fixed Haar measure $\mu$ on $A$ such $\beta^{u}=\mu$ for all $u \in \mathcal{G}^{(0)}$.

If $\chi \in \hat{A}$, then we get a $\mathcal{G}$-equivariant map $f^{\chi}: \mathcal{G}^{(0)} \times A \rightarrow \mathcal{G}^{(0)} \times \mathbf{T}$ given by $f^{\chi}(u, a)=(u, \chi(a))$. Thus we can form the pushout $f_{*}^{\chi}(\Sigma)$ so that

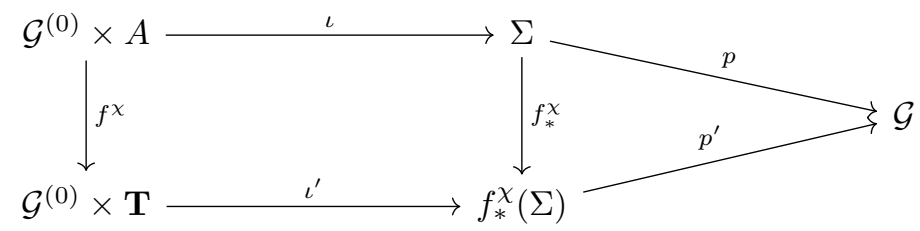

commutes. Then $C^{*}\left(\mathcal{G} ; f_{*}^{\chi}(\Sigma)\right)$ is the completion of $C_{c}^{\chi}(\Sigma)$ consisting of functions $g \in C_{c}(\Sigma)$ such that $g(\iota(r(\sigma), a) \sigma)=\chi(a) g(\sigma)$ with the *-algebra structure discussed at the beginning of this section.

Proposition 3.6. Let $\Sigma$ be a generalized twist as in Example 3.5. For $\chi \in \hat{A}$, let $f^{\chi}: \mathcal{G}^{(0)} \times A \rightarrow \mathcal{G}^{(0)} \times \mathbf{T}$ and $f_{*}^{\chi}(\Sigma)$ be the $\mathcal{G}$-equivariant map and $\mathbf{T}$-groupoid defined above. Then with notation as above,

$$
C^{*}(\Sigma) \cong C^{*}\left(\hat{A} \times \mathcal{G} ; f_{*}(\hat{A} \times \Sigma)\right)
$$

and $C^{*}(\Sigma)$ is the section algebra of an upper-semicontinuous $C^{*}$-bundle over $\hat{A}$ with fiber at $\chi \in \hat{A}$ isomorphic to $C^{*}\left(\mathcal{G} ; f_{*}^{\chi}(\Sigma)\right)$.

Proof. The isomorphism in (3.3) comes from Theorem 3.2.

The map $p: \hat{A} \times \mathcal{G}^{(0)} \rightarrow \mathcal{G}^{(0)}$ is continuous and satisfies $p \circ s=p \circ r$ so that $f_{*}(\hat{A} \times \Sigma)$ is a groupoid bundle over $\hat{A}$ as in Appendix A. Hence we can invoke Proposition A.1 to see that $C^{*}\left(\hat{A} \times \mathcal{G} ; f_{*}(\hat{A} \times \Sigma)\right)$ is isomorphic to the section algebra of an upper-semicontinuous $C^{*}$-bundle over $\hat{A}$. Since we can identify $f_{*}(\hat{A} \times \Sigma)(\chi)$ with $f_{*}^{\chi}(\Sigma)$ and $(\hat{A} \times \mathcal{G})(\chi)$ with $\mathcal{G}$, the result follows.

Proposition 3.7. With notation as in Example 3.5, suppose that $A$ compact. Then the dual $\hat{A}$ is discrete and

$$
C^{*}(\Sigma) \cong \bigoplus_{\chi \in \hat{A}} C^{*}\left(\mathcal{G} ; f_{*}^{\chi}(\Sigma)\right) \quad \text { and } \quad C_{r}^{*}(\Sigma) \cong \bigoplus_{\chi \in \hat{A}} C_{r}^{*}\left(\mathcal{G} ; f_{*}^{\chi}(\Sigma)\right)
$$

Proof. To prove the first isomorphism, note that by Proposition A.1

$$
C^{*}(\Sigma) \cong C^{*}\left(\hat{A} \times \mathcal{G} ; f_{*}(\hat{A} \times \Sigma)\right)
$$

is a $C_{0}(\hat{A})$-algebra. That is, letting $Z M\left(C^{*}(\Sigma)\right)$ denote the center of $M\left(C^{*}(\Sigma)\right)$, there is a $\sigma$-unital *-homomorphism $\rho: C_{0}(\hat{A}) \rightarrow Z M\left(C^{*}(\Sigma)\right)$. Since $\hat{A}$ is discrete, the images of the characteristic functions of singleton sets under $\rho$ give rise to a family $\left\{q_{\chi}\right\}_{\chi \in \hat{\mathcal{A}}}$ of mutually orthogonal central projections in $M\left(C^{*}(\Sigma)\right)$ which sum to unity in the strict topology. Moreover, the summands coincide with the fibers of the upper-semicontinuous $C^{*}$-bundle over $\hat{A}$ given in Proposition 3.6 and hence

$$
q_{\chi} C^{*}(\Sigma) q_{\chi}=q_{\chi} C^{*}(\Sigma) \cong C^{*}\left(\mathcal{G} ; f_{*}^{\chi}(\Sigma)\right) .
$$

for all $\chi \in \hat{A}$. 
For the second isomorphism, let $\pi: C^{*}(\Sigma) \rightarrow C_{r}^{*}(\Sigma)$ be the canonical quotient map. An argument like that of the preceding paragraph using the family $\left\{\pi\left(q_{\chi}\right)\right\}_{\chi \in \hat{\mathcal{A}}}$ of mutually orthogonal central projections in $M\left(C_{r}^{*}(\Sigma)\right)$ gives $C_{r}^{*}(\Sigma) \cong$ $\bigoplus_{\chi \in \hat{\mathcal{A}}} \pi\left(q_{\chi}\right) C_{r}^{*}(\Sigma)$. Lemma 3.1 gives $\pi\left(q_{\chi}\right) C_{r}^{*}(\Sigma) \cong C_{r}^{*}\left(\mathcal{G} ; f_{*}^{\chi}(\Sigma)\right)$, and the result follows.

Remark 3.8. If $A=\mathbf{T}$ and $\Sigma$ is a twist, then $\hat{A}=\mathbf{Z}$, and we have $\left[f_{*}^{n}(\Sigma)\right]=n[\Sigma]$ for $n \in \mathbf{Z}$. It follows that the central summand corresponding to $n=1$ is isomorphic to $C^{*}(\mathcal{G} ; \Sigma)$ and thus there is central projection $q=q_{1} \in M\left(C^{*}(\Sigma)\right)$ such that

$$
C^{*}(\mathcal{G} ; \Sigma) \cong q C^{*}(\Sigma) \text { and } C_{r}^{*}(G ; \Sigma) \cong q C_{r}^{*}(\Sigma)
$$

Now suppose that $\mathcal{G}=\mathcal{G}^{(0)}$ so that $\Sigma=\mathcal{A}$ is itself an abelian group bundle regarded as a groupoid with unit space $\mathcal{G}^{(0)}$ and let $\Lambda$ be a $\mathbf{T}$-twist over $\mathcal{A}$. Then since $\mathcal{A}$ is amenable $C^{*}(\mathcal{A} ; \Lambda)=C_{r}^{*}(\mathcal{A} ; \Lambda)$ (see, for example [SW13, Thm 1]). We shall say that such a twist is abelian if $\Lambda$ is also an abelian group bundle - that is if $\Lambda(u)$ is abelian for each $u \in \mathcal{G}^{(0)}$. Then $\Lambda$ is abelian if and only if $C^{*}(\Lambda)$ is abelian and in that case $C^{*}(\Lambda) \cong C_{0}(\hat{\Lambda})$. Arguing as in Example 3.4, we see that such extensions must be pointwise trivial but need not be globally trivial. If $\Lambda$ is determined by a continuous $\mathbf{T}$-valued 2-cocycle $c$, then $\Lambda$ is abelian if and only if $c$ is symmetric (cf., $\left[\mathrm{DGN}^{+} 20\right.$, Lemma 3.5]). Suppose now that $\Lambda$ is abelian. For $n \in \mathbf{Z}$, let $V_{n}:=\left\{\chi \in \hat{\Lambda}: \chi(z, u)=z^{n}\right.$ for all $z \in \mathbf{T}$ and $\left.u \in \mathcal{G}^{(0)}\right\}$. Then $C^{*}(\Lambda) \cong C_{0}(\hat{\Lambda})$ decomposes as a direct sum with summands of the form $C_{0}\left(V_{n}\right)$. Note that each $V_{n}$ is clopen. The projection $q$ in Remark 3.8 may then be identified with the characteristic function of $U_{\Lambda}:=V_{1}$ and hence

$$
C^{*}(\mathcal{A} ; \Lambda) \cong q C^{*}(\Lambda) \cong C_{0}\left(U_{\Lambda}\right) .
$$

See [DGN20, Section 3] for a related construction.

In the case that $\Lambda \cong \mathbf{T} \times \mathcal{A}$ and thus $\hat{\Lambda} \cong \mathbf{Z} \times \hat{\mathcal{A}}$, we have $U_{\Lambda} \cong\{1\} \times \hat{\mathcal{A}} \cong \hat{\mathcal{A}}$.

We return now to the more general situation where $\Sigma$ is a unit space fixing extension of $\mathcal{G}$ by the group bundle $\mathcal{A}$ as in the diagram ( $\dagger$ ) from the introduction. Suppose that, in addition, $\Omega$ is a $\mathbf{T}$-groupoid extension of $\Sigma$

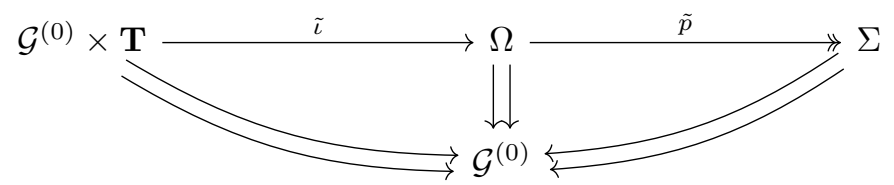

such that $\Lambda_{\Omega}:=\tilde{p}^{-1}(\mathcal{A})$, its restriction to $\mathcal{A}$, is an abelian group bundle over $\mathcal{G}^{(0)}$. We may thus regard $\Omega$ as an extension of $\mathcal{G}$ by $\Lambda_{\Omega}$. We assume that $\mathcal{A}, \Sigma$ and $\mathcal{G}$ are endowed with Haar systems that satisfy (3.1), the Haar system in $\mathcal{G}^{(0)} \times \mathbf{T}$ is given by the Haar measure on $\mathbf{T}$, and the Haar system on $\Omega$ is the one naturally defined by the Haar systems on $\mathcal{G}^{(0)} \times \mathbf{T}$ and $\Sigma$. To declutter notation a little, we write $\widehat{\Lambda}_{\Omega}$ for the dual bundle $\left(\Lambda_{\Omega}\right)^{\wedge}$.

Corollary 3.9. With notation as above let $f: \widehat{\Lambda}_{\Omega} * \Lambda_{\Omega} \rightarrow \widehat{\Lambda}_{\Omega} \times \mathbf{T}$ be given by $f(\chi, a)=(\chi, \chi(a))$. Then

$$
\begin{aligned}
& C^{*}(\Omega) \cong C^{*}\left(\widehat{\Lambda}_{\Omega} \rtimes \mathcal{G} ; f_{*}\left(\widehat{\Lambda}_{\Omega} \rtimes \Omega\right)\right) \quad \text { and } \\
& C_{r}^{*}(\Omega) \cong C_{r}^{*}\left(\widehat{\Lambda}_{\Omega} \rtimes \mathcal{G} ; f_{*}\left(\widehat{\Lambda}_{\Omega} \rtimes \Omega\right)\right) .
\end{aligned}
$$


Proof. This follows immediately from Remark 3.8, the above discussion, and Theorem 3.2 with $\Lambda_{\Omega}$ in place of $\mathcal{A}$.

By arguing as in Remark 3.8 and Corollary 3.9 we may conclude that $C^{*}(\Sigma ; \Omega)$ is isomorphic to the corner associated to the central projection $q_{\Omega}$ in

$$
M\left(C^{*}\left(\widehat{\Lambda}_{\Omega} \rtimes \mathcal{G} ; f_{*}\left(\widehat{\Lambda}_{\Omega} \rtimes \Omega\right)\right)\right)
$$

corresponding to the characteristic function of

$$
U_{\Omega}:=U_{\Lambda_{\Omega}} \subset \widehat{\Lambda}_{\Omega}=\left(\widehat{\Lambda}_{\Omega} \rtimes \mathcal{G}\right)^{(0)} .
$$

Observe that $U_{\Omega}$ is an invariant clopen set under the action of both $\mathcal{G}$ and $\Omega$ and thus both groupoids act on $U_{\Omega}$.

Corollary 3.10. With notation as above define $g: U_{\Omega} * \Lambda_{\Omega} \rightarrow U_{\Omega} \times \mathbf{T}$ by $g(\chi, a)=$ $(\chi, \chi(a))$. Then

$C^{*}(\Sigma ; \Omega) \cong C^{*}\left(U_{\Omega} \rtimes \mathcal{G} ; g_{*}\left(U_{\Omega} \rtimes \Omega\right)\right) \quad$ and $\quad C_{r}^{*}(\Sigma ; \Omega) \cong C_{r}^{*}\left(U_{\Omega} \rtimes \mathcal{G} ; g_{*}\left(U_{\Omega} \rtimes \Omega\right)\right)$.

Proof. Observe that

$$
\left(\widehat{\Lambda}_{\Omega} \rtimes \mathcal{G}\right)_{U_{\Omega}} \cong U_{\Omega} \rtimes \mathcal{G} \text { and }\left(\widehat{\Lambda}_{\Omega} \rtimes \Omega\right)_{U_{\Omega}} \cong U_{\Omega} \rtimes \Omega .
$$

For $(\chi, a) \in U_{\Omega} * \Lambda_{\Omega} \subset \widehat{\Lambda}_{\Omega} * \Lambda_{\Omega}$,

$$
f(\chi, a)=(\chi, \chi(a))=g(\chi, a) \in U_{\Omega} \times \mathbf{T}
$$

Therefore,

$$
\left(f_{*}\left(\widehat{\Lambda}_{\Omega} \rtimes \Omega\right)\right)_{U_{\Omega}} \cong g_{*}\left(U_{\Omega} \rtimes \Omega\right) .
$$

Hence, by Remark 3.8 and Corollary 3.9

$$
\begin{aligned}
C^{*}(\Sigma ; \Omega) & \cong q_{\Omega} C^{*}\left(\widehat{\Lambda}_{\Omega} \rtimes \mathcal{G} ; f_{*}\left(\widehat{\Lambda}_{\Omega} \rtimes \Omega\right)\right) q_{\Omega} \\
& \cong C^{*}\left(\left(\widehat{\Lambda}_{\Omega} \rtimes \mathcal{G}\right)_{U_{\Omega}} ;\left(f_{*}\left(\widehat{\Lambda}_{\Omega} \rtimes \Omega\right)\right)_{U_{\Omega}}\right) \\
& \cong C^{*}\left(U_{\Omega} \rtimes \mathcal{G} ; g_{*}\left(U_{\Omega} \rtimes \Omega\right)\right) .
\end{aligned}
$$

The case for the reduced $C^{*}$-algebras follows by a similar argument.

Recall that an étale groupoid $\mathcal{G}$ is said to be effective if the interior of the isotropy groupoid is $\mathcal{G}^{(0)}$ and topologically principal if the set of points with trivial isotropy is dense in $\mathcal{G}^{(0)}$. These notions are equivalent if the étale groupoid $\mathcal{G}$ is second countable (see [BCFS14, Lemma 3.1]). The above corollary allows us to generalize $\left[\mathrm{IKR}^{+} 21\right.$, Theorem 4.6] (see also $\left[\mathrm{DGN}^{+}\right.$20, Theorem 5.8] and [DGN20, Theorem 4.6]).

Corollary 3.11. With notation as above, suppose that $\mathcal{G}$ is étale and that the action groupoid $U_{\Omega} \rtimes \mathcal{G}$ is second countable and effective. Then the image of $C_{r}^{*}\left(\mathcal{A}, \Lambda_{\Omega}\right)$ under the natural embedding into $C_{r}^{*}(\Sigma ; \Omega)$ is a Cartan subalgebra with Weyl twist $g_{*}\left(U_{\Omega} \rtimes \Omega\right)$.

Proof. This follows from Corollary 3.10 and [Ren08, Theorem 5.2]. 
Example 3.12. Let $H$ be a discrete abelian group and let $E$ be a $\mathbf{T}$-twist over $H$-that is, a central extension by $\mathbf{T}$. Since $H$ is discrete, there is a $\mathbf{T}$-valued skew-symmetric bicharacter $\varpi$ on $H$ and a set of generating unitaries $\left\{u_{h} \mid h \in H\right\}$ in $C^{*}(H ; E)$ such that for all $g, h \in H$

$$
u_{g} u_{h}=\varpi(g, h) u_{h} u_{g} .
$$

By [Kle65, Lemma 7.2] the extension $E$ is trivial if and only if $\varpi(g, h)=1$ for all $g, h \in H$. Let $A$ be a subgroup of $H$ which is maximal amongst subgroups on which $\varpi(\cdot, \cdot)$ is identically 1. It is shown in [Kum86, Example 1.12] that the $C^{*}$-subalgebra $B$ generated by $\left\{u_{a} \mid a \in A\right\}$ is a diagonal subalgebra of $C^{*}(H ; E)$. We now show that this also follows from Corollary 3.11 with $\Sigma:=H, \mathcal{A}:=A$, $\mathcal{G}=H / A$ and $\Omega:=E$.

Since the restriction of $\varpi$ to $A$ is trivial the extension $E$ is trivial on $A$ and thus $\Lambda$ is trivial as a $\mathbf{T}$-twist. Hence, $B \cong C^{*}(A)$ and $U_{\Lambda} \cong \hat{A}$. There is a continuous homomorphism $\varpi_{A}: H \rightarrow \hat{A}$ such that for all $h \in H, a \in A$

$$
\left(\varpi_{A}(h)\right)(a)=\varpi(h, a) .
$$

Moreover, $A=\operatorname{ker} \varpi$ and thus $\varpi$ induces an injection $H / A \rightarrow \hat{A}$. The action of $H / A$ on $\hat{A}$ is then given by translation and, hence, is free. Since $H / A$ is étale and its action on $U_{\Omega} \cong \hat{A}$ is principal, the image of $C_{r}^{*}\left(\mathcal{A}, \Lambda_{\Omega}\right) \cong C^{*}(A)$ under the natural embedding into $C_{r}^{*}(\Sigma ; \Omega)=C^{*}(H ; E)$ is a diagonal subalgebra.

3.2. Extensions by 2-cocycles. Extensions associated to groupoid 2-cocycles yield some nice applications of the pushout construction. For convenience, we review the basics here. (For more details, see [IKSW19, Appendix A].) Assume that $p_{\mathcal{A}}: \mathcal{A} \rightarrow \mathcal{G}^{(0)}$ is a $\mathcal{G}$-bundle. As before we write $\mathcal{A}(u)$ for $p_{\mathcal{A}}^{-1}(u)$ for $u \in \mathcal{G}^{(0)}$. Assume that $\varphi: \mathcal{G}^{(2)} \rightarrow \mathcal{A}$ is a continuous normalized 2-cocycle. That is, $\varphi\left(\gamma_{1}, \gamma_{2}\right) \in$ $\mathcal{A}\left(r\left(\gamma_{1}\right)\right)$ for all $\left(\gamma_{1}, \gamma_{2}\right) \in \mathcal{G}^{(2)}, \varphi\left(\gamma_{0}, \gamma_{1}\right)+\varphi\left(\gamma_{0} \gamma_{1}, \gamma_{2}\right)=\gamma_{0} \cdot \varphi\left(\gamma_{1}, \gamma_{2}\right)+\varphi\left(\gamma_{0}, \gamma_{1} \gamma_{2}\right)$ for all $\left(\gamma_{0}, \gamma_{1}\right),\left(\gamma_{1}, \gamma_{2}\right) \in \mathcal{G}^{(2)}$, and $\varphi(\gamma, u)=\varphi(u, \gamma)=0_{u}$ for all $\gamma \in \mathcal{A}(u)$ and $u \in \mathcal{G}^{(0)}$. Then the extension $\Sigma_{\varphi}$ of $\mathcal{G}$ by $\mathcal{A}$ determined by $\varphi$ is obtained by giving the fibered product $\mathcal{A} * \mathcal{G}$ the groupoid structure where $\left(a_{1}, \gamma_{1}\right)\left(a_{2}, \gamma_{2}\right)=\left(a_{1}+\gamma_{1}\right.$. $\left.a_{2}+\varphi\left(\gamma_{1}, \gamma_{2}\right), \gamma_{1} \gamma_{2}\right)$ if $\left(\gamma_{1}, \gamma_{2}\right) \in \mathcal{G}^{(2)}$ and $(a, \gamma)^{-1}=\left(-\gamma^{-1} \cdot a-\varphi\left(\gamma^{-1}, \gamma\right), \gamma^{-1}\right)$. We exhibit $\Sigma_{\varphi}$ as an extension of $\mathcal{G}$ by $\mathcal{A}$ via $i(a)=\left(a, p_{\mathcal{A}}(a)\right)$ and $p(a, \gamma)=\gamma$.

Example 3.13. If $\mathcal{A}=\mathcal{G}^{(0)} \times A$ is the trivial bundle (with trivial action), then an $\mathcal{A}$-valued cocycle is given by a continuous $A$-valued 2-cocycle $\sigma$ on $\mathcal{G}$ via the formula $\varphi\left(\gamma_{1}, \gamma_{2}\right)=\left(\sigma\left(\gamma_{1}, \gamma_{2}\right), r\left(\gamma_{1}\right)\right)$.

Example 3.14. Let $\varphi$ be a continuous normalized $\mathbf{T}$-valued 2 -cocycle and let $\Sigma_{\varphi}$ be the $\mathbf{T}$-twist associated to $\varphi$. Then by Proposition 3.7 and Remark 3.8, and the fact that $\Sigma_{\varphi^{n}} \cong n_{*}\left(\Sigma_{\varphi}\right)$ for all $n \in \mathbf{Z}$, we have

$$
C^{*}\left(\Sigma_{\varphi}\right) \cong \bigoplus_{n \in \mathbf{Z}} C^{*}\left(\mathcal{G} ; \Sigma_{\varphi^{n}}\right) .
$$

This recovers [BaH14, Theorem 3.2].

Example 3.15 (Transformation groupoids). Let $\mathcal{G}$ be a groupoid acting on the right of a locally compact Hausdorff space $X$. Recall that the transformation groupoid $X \rtimes \mathcal{G}$ is obtained by endowing the fibered product $X * \mathcal{G}$ with the groupoid operations $\left(x, \gamma_{1}\right)\left(x \cdot \gamma_{1}, \gamma_{2}\right)=\left(x, \gamma_{1} \gamma_{2}\right)$ if $\left(\gamma_{1}, \gamma_{2}\right) \in \mathcal{G}^{(2)}$ and $(x, \gamma)^{-1}=\left(x \cdot \gamma, \gamma^{-1}\right)$. 
Assume that $\varphi: \mathcal{G}^{(2)} \rightarrow \mathcal{A}$ is a 2-cocycle as above. Then one can define a natural 2-cocycle $\tilde{\varphi}:(X \rtimes \mathcal{G})^{(2)} \rightarrow X * \mathcal{A}$ via $\tilde{\varphi}\left(\left(x, \gamma_{1}\right),\left(x \cdot \gamma_{1}, \gamma_{2}\right)\right)=\left(x, \varphi\left(\gamma_{1}, \gamma_{2}\right)\right)$. The extension $\Sigma_{\tilde{\varphi}}$ of $X \rtimes \mathcal{G}$ defined by $\tilde{\varphi}$ is isomorphic to the extension $X \rtimes \Sigma_{\varphi}$, where $\Sigma_{\varphi}$ is the extension of $\mathcal{G}$ defined by $\varphi$. To see this, note that $\Sigma_{\tilde{\varphi}}=\{((x, a),(x, \gamma))$ : $\left.x \in X, a \in \mathcal{A}^{x}, \gamma \in \mathcal{G}^{x}\right\}$ with the operations

$$
\left(\left(x, a_{1}\right),\left(x, \gamma_{1}\right)\right)\left(\left(x \cdot \gamma_{1}, a_{2}\right),\left(x \cdot \gamma_{1}, \gamma_{2}\right)\right)=\left(\left(x, a_{1}+\gamma_{1} a_{2}+\varphi\left(\gamma_{1}, \gamma_{2}\right)\right),\left(x, \gamma_{1} \gamma_{2}\right)\right)
$$

and

$$
((x, a),(x, \gamma))^{-1}=\left(\left(x \cdot \gamma,-\gamma^{-1} a-\varphi\left(\gamma^{-1}, \gamma\right)\right),\left(x \cdot \gamma, \gamma^{-1}\right)\right) .
$$

On the other hand, $X \rtimes \Sigma_{\varphi}=\left\{(x,(a, \gamma)): x \in X, a \in \mathcal{A}^{x}, \gamma \in \mathcal{G}^{x}\right\}$ with the operations

$$
\left(x,\left(a_{1}, \gamma_{1}\right)\right)\left(x \cdot \gamma_{1},\left(a_{2}, \gamma_{2}\right)\right)=\left(x,\left(a_{1}+\gamma_{1} a_{2}+\varphi\left(\gamma_{1}, \gamma_{2}\right), \gamma_{1} \gamma_{2}\right)\right)
$$

and

$$
(x,(a, \gamma))^{-1}=\left(x \cdot \gamma,\left(-\gamma^{-1} \cdot a-\varphi\left(\gamma^{-1}, \gamma\right), \gamma^{-1}\right)\right) .
$$

Therefore the map $V: \Sigma_{\tilde{\varphi}} \rightarrow X \rtimes \Sigma_{\varphi}$ defined by $V((x, a),(x, \gamma))=(x,(a, \gamma))$ is a groupoid isomorphism.

Suppose that $p_{\mathcal{B}}: \mathcal{B} \rightarrow \mathcal{G}^{(0)}$ is another abelian $\mathcal{G}$-bundle and that $f: \mathcal{A} \rightarrow$ $\mathcal{B}$ is an equivariant map such that $\left.f\right|_{\mathcal{A}(u)}: \mathcal{A}(u) \rightarrow \mathcal{B}(u)$ is a continuous group homomorphism for all $u \in \mathcal{G}^{(0)}$. There is a $\mathcal{B}$-valued 2-cocycle $f_{*}(\varphi): \mathcal{G}^{(2)} \rightarrow \mathcal{B}$ given by $f_{*}(\varphi)\left(\gamma_{1}, \gamma_{2}\right)=f\left(\varphi\left(\gamma_{1}, \gamma_{2}\right)\right)$.

Lemma 3.16. Let $\Sigma_{f_{*}(\varphi)}$ be the extension of $\mathcal{G}$ by $\mathcal{B}$ determined by $f_{*}(\varphi)$. Then $f_{*} \Sigma_{\varphi}$ is properly isomorphic to $\Sigma_{f_{*}(\varphi)}$.

Proof. Define $g: \Sigma_{\varphi} \rightarrow \Sigma_{f_{*}}$ by $g(a, \gamma)=(f(a), \gamma)$. The diagram

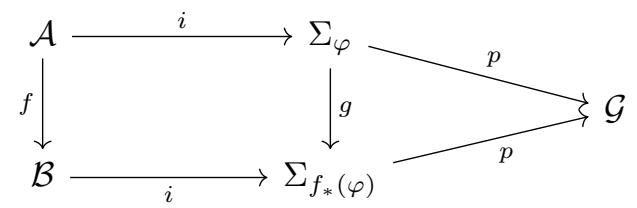

commutes. Therefore the lemma follows from Theorem 1.5.

3.3. The T-groupoid defined by a 2-cocycle. We continue to assume the setting from Section 3.2: $\mathcal{A}$ is an abelian $\mathcal{G}$-bundle, $\varphi: \mathcal{G}^{(2)} \rightarrow \mathcal{A}$ is a 2-cocycle, and $\Sigma_{\varphi}$ is the extension defined by $\varphi$. Then, as in Example 3.15 there is a 2-cocycle

$$
\tilde{\varphi}:(\hat{\mathcal{A}} \rtimes \mathcal{G})^{(2)} \rightarrow \hat{\mathcal{A}} * \mathcal{A}
$$

defined by

$$
\tilde{\varphi}\left(\left(\chi, \gamma_{1}\right),\left(\chi \cdot \gamma_{1}, \gamma_{2}\right)\right)=\left(\chi, \varphi\left(\gamma_{1}, \gamma_{2}\right)\right)
$$

if $\left(\gamma_{1}, \gamma_{2}\right) \in \mathcal{G}^{(2)}$. Therefore we can identify $\hat{\mathcal{A}} \rtimes \Sigma_{\varphi}$ with $\Sigma_{\tilde{\varphi}}$, the extension of $\hat{\mathcal{A}} \rtimes \mathcal{G}$ determined by $\tilde{\varphi}$. Consider the 2-cocycle $\hat{\varphi}:=f_{*} \tilde{\varphi}:(\hat{\mathcal{A}} \rtimes \mathcal{G})^{(2)} \rightarrow \hat{\mathcal{A}} \times \mathbf{T}$ defined via

$$
\hat{\varphi}\left(\left(\chi, \gamma_{1}\right),\left(\chi, \gamma_{2}\right)\right)=\left(\chi, \chi\left(\varphi\left(\gamma_{1}, \gamma_{2}\right)\right)\right) .
$$

Lemma 3.16 and Theorem 3.2 imply that $\widetilde{\Sigma}_{\varphi}$ is isomorphic to the $\mathbf{T}$-groupoid defined by $\hat{\varphi}$ and $C^{*}\left(\Sigma_{\varphi}\right)$ is isomorphic to $C^{*}\left(\hat{\mathcal{A}} \rtimes \mathcal{G} ; \Sigma_{\hat{\varphi}}\right)$. 
Example 3.17. The following example was studied in [IKSW19]. Let $X$ be a secondcountable locally compact Hausdorff space, and $G$ a second-countable locally compact abelian group. Let $\mathscr{G}$ denote the sheaf of germs of continuous $G$-valued functions on $X$, and let $c \in Z^{2}(\mathscr{U}, \mathscr{G})$ be a normalized Cech two cocycle for some locally finite cover $\mathscr{U}=\left\{U_{i}\right\}_{i \in I}$ of $X$ by precompact open sets. The blow-up groupoid $\mathcal{G}_{\mathscr{U}}$ with respect to the natural map from $\bigsqcup_{i} U_{i}$ into $X$ is

$$
\mathcal{G}_{\mathscr{U}}=\left\{(i, x, j): x \in U_{i j}:=U_{i} \cap U_{j}\right\}
$$

with $(i, x, j)(j, x, k)=(i, x, k)$ and $(i, x, j)^{-1}=(j, x, i)$. As noted in [IKSW19, Remark 3.3], the Čech 2-cocycle $c$ defines a groupoid 2-cocycle $\varphi_{c}: \mathcal{G}_{\mathscr{\mathscr { U }}}^{(2)} \rightarrow G$ via

$$
\varphi_{c}((i, x, j),(j, x, k))=c_{i j k}(x) .
$$

Let $\Sigma_{c}$ be the extension of $\mathcal{G}_{\mathscr{U}}$ by the 2-cocycle $\varphi_{c}$. Define

by

$$
\hat{\varphi}:\left(\left(\hat{G} \times \bigsqcup_{i} U_{i}\right) \rtimes \mathcal{G}_{\mathscr{U}}\right)^{(2)} \rightarrow \mathbf{T} \times \hat{G} \times \bigsqcup_{i} U_{i}
$$

$$
\hat{\varphi}((\tau,(i, x, j)),(\tau,(j, x, k)))=\left(\overline{\tau\left(c_{i j k}(x)\right)}, \tau\right)
$$

for $\tau \in \hat{G}$ and $((i, x, j),(j, x, k)) \in\left(\mathcal{G}_{\mathscr{U}}\right)^{(2)}$. Then $\hat{\varphi}$ is a groupoid 2-cocycle, and the pushout groupoid $\widetilde{\Sigma}$ is isomorphic to the $\mathbf{T}$-groupoid that is the extension of $\left(\hat{G} \times \bigsqcup_{i} U_{i}\right) \rtimes \mathcal{G}_{\mathscr{U}}$ defined by $\hat{\varphi}$.

Let $\mathscr{V}=\left\{\hat{G} \times U_{i}\right\}_{i \in I}$ be the locally finite cover of $\hat{G} \times X$, let $\mathscr{S}$ be the sheaf of germs of continuous $\mathbf{T}$-valued functions, and define $\nu^{c}=\left\{\nu_{i j k}^{c}\right\} \in Z^{2}(\mathscr{V}, \mathscr{S})$ by

$$
\nu^{c}((\tau,(i, x, j)),(\tau,(j, x, k)))=\overline{\tau\left(c_{i j k}(x)\right)} .
$$

Then the 2-cocycle $\hat{\varphi}$ is defined by the Čech 2-cocycle $\nu^{c} \in Z^{2}(\mathscr{V}, \mathscr{S})$.

That is, $\nu^{c}$ is the normalized 2-cocycle considered in [IKSW19, Equation (3.4)]. Hence the generalized Raeburn-Taylor $C^{*}$-algebra $A(\nu)$ studied in [IKSW19] is isomorphic to the restricted $C^{*}$-algebra of the $\mathbf{T}$-groupoid defined by the 2 -cocycle $\nu^{c}$.

By [IKSW19, Lemma 5.2], $A(\nu)$ is a continuous-trace $C^{*}$-algebra with spectrum $\hat{G} \times X$ with Dixmier-Douady invariant $\delta(A(\nu))=\left[\nu^{c}\right]$. For a concrete example, let $G=\mathbf{Z}$ and choose a Čech 2-cocycle $c$ associated to any line bundle.

Example 3.18. This example is an expansion of $\left[\mathrm{IKR}^{+} 21\right.$, Example 4.10]. Let $\Gamma=\mathbf{Z}$ act on $\mathbf{T}$ via rotation by $\alpha \in \mathbf{Q}: z \cdot k:=z e^{2 \pi i k \alpha}$. If $\alpha=m / n$ with $m$ and $n$ relatively prime, then $n \mathbf{Z}$ fixes the action. We have a short exact sequence of groups

$$
n \mathbf{Z} \longrightarrow \mathbf{Z} \stackrel{p}{\longrightarrow} \mathbf{Z}_{n} \text {. }
$$

The action on $\mathbf{T}$ leads to an extension of groupoids

$$
n \mathbf{Z} \times \mathbf{T} \stackrel{i}{\longrightarrow} \mathbf{T} \rtimes \mathbf{Z} \stackrel{\pi}{\longrightarrow} \mathbf{T} \rtimes \mathbf{Z}_{n} .
$$

Thus, using the notation from the previous section, $\mathcal{A}=\mathbf{T} \times n \mathbf{Z}, \Sigma=\mathbf{T} \rtimes \mathbf{Z}$, and $\mathcal{G}=\mathbf{T} \rtimes \mathbf{Z}_{n}$. The $C^{*}$-algebra $C^{*}(\mathbf{T} \rtimes \mathbf{Z})$ is the rational rotation $C^{*}$-algebra $\mathcal{A}_{\alpha}$ (see, for example, [DB84]). The groupoid $\mathcal{D}$ is the cartesian product $\mathbf{T} \times \mathbf{T}_{n} \times$ $\mathbf{T} \times \mathbf{Z}$, where $\mathbf{T}_{n}=\mathbf{T} / \mathbf{Z}_{n}$ is the dual of $n \mathbf{Z}$. The extension $\widetilde{\Sigma}$ is the quotient of $\mathcal{D}$ where we identify $(\omega, \chi, z, n l+k)$ with $\left(\omega, \chi^{n l}, z, k\right)$. Therefore the rational rotation 
algebra $\mathcal{A}_{\alpha}$ is the completion of continuous functions $F$ on $\mathbf{T} \times \mathbf{T}_{n} \times \mathbf{Z}$ such that $F(\omega, \chi, n l+k)=\chi^{n l} F(\omega, \chi, k)$ for all $l \in \mathbf{Z}$.

The extension $\widetilde{\Sigma}$ is properly isomorphic to the one defined by a 2-cocycle. Indeed, let $\sigma=e^{2 \pi i \alpha} \in \mathbf{T}$ and view $\sigma$ as a character on $\mathbf{Z}$. Thus we can identify $\mathbf{Z}_{n}$ with $\sigma(\mathbf{Z})$ and then the map $p$ in the short exact sequence (3.5) equals $\sigma$. Choose $s \in \mathbf{Z}$ such that $s m=1(\bmod n)$. Then the map $\tau: \mathbf{Z}_{n} \rightarrow \mathbf{Z}$ defined by $\tau(k)=s k$ defines a cross-section of $\sigma$. In particular, $\mathbf{Z}$ is properly isomorphic to the extension $n \mathbf{Z} \times{ }_{\omega} \mathbf{Z}_{n}$ by a two cocycle $\omega: \mathbf{Z}_{n} \times \mathbf{Z}_{n} \rightarrow n \mathbf{Z}$ defined by $\tau$. Using the proof of [IKSW19, Proposition A.6], $\omega\left(\dot{k}_{1}, \dot{k}_{2}\right)=\tau\left(\dot{k}_{1}\right)+\tau\left(\dot{k}_{2}\right)-\tau\left(\dot{k}_{1}+\dot{k}_{2}\right)$. A quick computation shows that

$$
\omega\left(\dot{k}_{1}, \dot{k}_{2}\right)= \begin{cases}0 & \text { if } \dot{k}_{1}+\dot{k}_{2}<n \\ n s & \text { if } \dot{k}_{1}+\dot{k}_{2} \geq n\end{cases}
$$

which recovers the 2-cocycle used in Step 2 of the proof of [DB84, Proposition 1].

The map $\underline{\tau}: \mathbf{T} \rtimes \mathbf{Z}_{n} \rightarrow \mathbf{T} \rtimes \mathbf{Z}$ defined by $\underline{\tau}(z, k)=(z, \tau(k))$ is a crosssection of the extension of the groupoids (3.6). Hence $\mathbf{T} \rtimes \mathbf{Z}$ is properly isomorphic to the extension given by the 2-cocycle $\varphi \in Z^{2}\left(\mathbf{T} \rtimes \mathbf{Z}_{n}, \mathbf{T} \times n \mathbf{Z}\right)$ defined by $\varphi\left(\left(w, \dot{k}_{1}\right),\left(w \cdot \dot{k}_{1}, \dot{k}_{2}\right)\right)=\left(w, \omega\left(\dot{k}_{1}, \dot{k}_{2}\right)\right)$. The extension of the 2 -cocycle $\varphi$ is $\Sigma_{\varphi}=\mathbf{T} \times n \mathbf{Z} \times \mathbf{Z}_{n}$ with operations $\left(w, n l_{1}, \dot{k}_{1}\right)\left(w \cdot \dot{k}_{1}, n l_{2}, \dot{k}_{2}\right)=\left(w, n l_{1}+n l_{2}+\right.$ $\left.\omega\left(\dot{k}_{1}, \dot{k}_{2}\right), \dot{k}_{1}+\dot{k}_{2}\right)$ and $(w, n l, \dot{k})^{-1}=(w,-n l-\omega(-\dot{k}, \dot{k}),-\dot{k})$. Following the proof of [IKSW19, Proposition A.6] the isomorphism between $\Sigma_{\varphi}$ and $\mathbf{T} \rtimes \mathbf{Z}$ is given by $(w, n l, \dot{k}) \mapsto(w, n l+\tau(\dot{k}))$.

We have that $\hat{\mathcal{A}} \simeq \mathbf{T}_{n} \times \mathbf{T}$ and $\hat{\mathcal{A}} * \mathcal{A} \simeq \mathbf{T}_{n} \times \mathbf{T} \times n \mathbf{Z}$. The action of $\mathcal{G}=$ $\mathbf{T} \rtimes \mathbf{Z}_{n}$ on $\hat{\mathcal{A}}$ is given via $(\chi, w) \cdot(w, \dot{k})=(\chi, w \cdot k)=\left(\chi, w \sigma^{k}\right)$. Therefore we can identify $\hat{\mathcal{A}} \rtimes \mathcal{G}$ with $\mathbf{T}_{n} \times \mathbf{T} \rtimes \mathbf{Z}_{n}:=\left\{(\chi, w, \dot{k}) \in \mathbf{T}_{n} \times \mathbf{T} \times \mathbf{Z}_{n}\right\}$, where $\left(\chi, w, \dot{k}_{1}\right) \cdot\left(\chi, w \cdot k_{1}, \dot{k}_{2}\right)=\left(\chi, w, \dot{k}_{1}+\dot{k}_{2}\right)$ and $(\chi, w, \dot{k})^{-1}=(\chi, w \cdot k,-\dot{k})$. Thus the 2-cocycle $\tilde{\varphi}:\left(\mathbf{T}_{n} \times \mathbf{T} \rtimes \mathbf{Z}_{n}\right)^{(2)} \rightarrow \mathbf{T}_{n} \times \mathbf{T} \times n \mathbf{Z}$ of (3.4) is defined by

$$
\tilde{\varphi}\left(\left(\chi, w, \dot{k}_{1}\right),\left(\chi, w \cdot \dot{k}_{1}, \dot{k}_{2}\right)\right)=\left(\chi, w, \omega\left(\dot{k}_{1}, \dot{k}_{2}\right)\right) .
$$

By Lemma 3.16, $\widetilde{\Sigma}$ is properly isomorphic to the extension by the 2-cocycle $\hat{\varphi}$ which is the pushout of $\tilde{\varphi}$. Therefore $\hat{\varphi}:\left(\mathbf{T}_{n} \times \mathbf{T} \rtimes \mathbf{Z}_{n}\right)^{(2)} \rightarrow \mathbf{T}_{n} \times \mathbf{T} \times \mathbf{T}$ is defined by

$$
\hat{\varphi}\left(\left(\chi, w, \dot{k}_{1}\right),\left(\chi, w \cdot \dot{k}_{1}, \dot{k}_{2}\right)\right)=\left(\chi, w, \chi^{\omega\left(\dot{k}_{1}, \dot{k}_{2}\right)}\right) .
$$

Hence the rotation algebra $\mathcal{A}_{\alpha}$ is isomorphic to $C^{*}\left(\mathbf{T}_{n} \times \mathbf{T} \rtimes \mathbf{Z}_{n} ; \Sigma_{\hat{\varphi}}\right)$. For $\chi \in \mathbf{T}_{n}$, define $\chi_{*}(\varphi):\left(\mathbf{T} \rtimes Z_{n}\right)^{(2)} \rightarrow \mathbf{T}$ by

$$
\chi_{*}(\varphi)\left(\left(w, \dot{k}_{1}\right),\left(w \cdot \dot{k}_{1}, \dot{k}_{2}\right)=\left(w, \chi^{\omega\left(\dot{k}_{1}, \dot{k}_{2}\right)}\right) .\right.
$$

Then Proposition 3.6 implies that $\mathcal{A}_{\alpha}$ is the section algebra of an upper-semicontinuous $C^{*}$-bundle over $\mathbf{T}_{n}$ with fiber at $\chi \in \mathbf{T}_{n}$ isomorphic to $C^{*}\left(\mathbf{T} \rtimes Z_{n} ; \Sigma_{\chi_{*}(\varphi)}\right)$. 


\section{Appendix A. Bundles of Twists}

Let $\Sigma$ be a twist over $\mathcal{G}$. Alternatively, $\Sigma$ is a $\mathbf{T}$-groupoid so that we have the following diagram

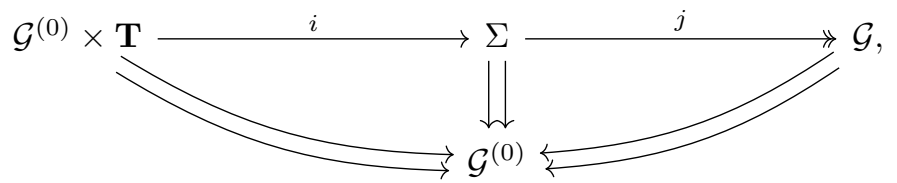

where as usual we have identified $\Sigma^{(0)}$ and $\mathcal{G}^{(0)}$. In particular, if $F \subset \mathcal{G}^{(0)}$ is $\mathcal{G}$-invariant, then it is $\Sigma$-invariant and the reduction $\left.\Sigma\right|_{F}$ is also a twist over the reduction $\left.\mathcal{G}\right|_{F}$.

Suppose that $p: \mathcal{G}^{(0)} \rightarrow T$ is a continuous map such that $p \circ r=r \circ s$. Then we say that $\Sigma$ is a groupoid bundle over $T$. ${ }^{1}$ Then $p^{-1}(t)$ is invariant for all $t \in T$. We write $\Sigma(t)$ and $\mathcal{G}(t)$ for the restrictions to $p^{-1}(t)$, respectively. Then $\Sigma(t)$ is a twist over $\mathcal{G}(t)$.

Proposition A.1. Suppose that $\mathcal{G}$ is a second countable locally compact Hausdorff groupoid with a Haar system and that $\Sigma$ is a twist over $\mathcal{G}$. If $p: \mathcal{G}^{(0)} \rightarrow T$ is a continuous map such that $p \circ r=p \circ s$, then $C^{*}(\mathcal{G} ; \Sigma)$ is a $C_{0}(T)$-algebra. Let $\Sigma(t)$ be the twist over $\mathcal{G}(t)$ defined above. Then $C^{*}(\mathcal{G} ; \Sigma)$ is (isomorphic to) the section algebra of an upper-semicontinuous $C^{*}$-bundle over $T$. The fibre $C^{*}(\mathcal{G} ; \Sigma)(t)$ is isomorphic to $C^{*}(\mathcal{G}(t) ; \Sigma(t))$.

Proof. Recall that $C^{*}(\mathcal{G} ; \Sigma)$ is the $C^{*}$-algebra $C^{*}(\mathcal{G}, \mathcal{B})$ of a Fell bundle $q: \mathcal{B} \rightarrow \mathcal{G}$ as described in [MW08, Example 2.9]. Similarly, $C^{*}(\mathcal{G}(t) ; \Sigma(t))$ is the $C^{*}$-algebra $C^{*}(\mathcal{G}(t), \mathcal{B})$ of $\left.q\right|_{q^{-1}(\mathcal{G}(t))}$. Let $U(t)=\mathcal{G}^{(0)} \backslash p^{-1}(t)$. Using [IW12, Theorem 3.7] (as in [SW13, Lemma 9]), we obtain a short exact sequence

$$
0 \longrightarrow C^{*}\left(\left.\mathcal{G}\right|_{U(t)}, \mathcal{B}\right) \stackrel{i}{\longrightarrow} C^{*}(\mathcal{G}, \mathcal{B}) \stackrel{j}{\longrightarrow} C^{*}(\mathcal{G}(t), \mathcal{B}) \longrightarrow 0
$$

where $i$ identifies $C^{*}\left(\left.\mathcal{G}\right|_{U(t)}, \mathcal{B}\right)$ with the completion in $C^{*}(\mathcal{G}, \mathcal{B})$ of the ideal of sections in $\Gamma_{c}(\mathcal{G}, \mathcal{B})$ that vanish off $\left.\mathcal{G}\right|_{U(t)}$, and $j$ is given on $\Gamma_{c}(\mathcal{G}, \mathcal{B})$ by restriction to $p^{-1}(t)$. Now exactly as in [Wil19, Proposition 5.37], we see that $C^{*}(\mathcal{G}, \mathcal{B})$ is a $C_{0}(T)$-algebra with fibres $C^{*}(\mathcal{G}, \mathcal{B})(t)$ identified with $C^{*}(\mathcal{G}(t), \mathcal{B})$.

\section{References}

[BaH14] Jonathan H. Brown and Astrid an Huef, Decomposing the $C^{*}$-algebras of groupoid extensions, Proc. Amer. Math. Soc. 142 (2014), 1261-1274.

[BCFS14] Jonathan H. Brown, Lisa Orloff Clark, Cynthia Farthing, and Aidan Sims, Simplicity of algebras associated to étale groupoids, Semigroup Forum 88 (2014), 433-452.

[DB84] Marc De Brabanter, The classification of rational rotation $C^{*}$-algebras, Arch. Math. (Basel) 43 (1984), 79-83.

[DGN20] Anna Duwenig, Elizabeth Gillaspy, and Rachael Norton, Analyzing the Weyl construction for dynamical Cartan subalgebras, preprint, 2020. arXiv:2010.04137 [math.OA].

$\left[\mathrm{DGN}^{+} 20\right]$ A. Duwenig, E. Gillaspy, R. Norton, S. Reznikoff, and S. Wright, Cartan subalgebras for non-principal twisted groupoid $C^{*}$-algebras, J. Funct. Anal. 279 (2020), 108611, 40 .

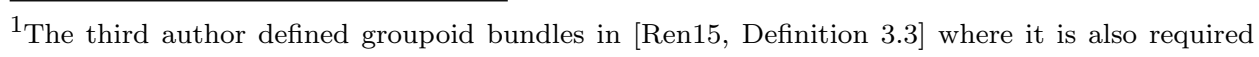
that $p$ be open. 
$\left[\mathrm{IKR}^{+}\right.$21] Marius Ionescu, Alex Kumjian, Jean N. Renault, Aidan Sims, and Dana P. Williams, $C^{*}$-algebras of extensions of groupoids by group bundles, J. Funct. Anal. 280 (2021), $108892,33$.

[IKSW19] Marius Ionescu, Alex Kumjian, Aidan Sims, and Dana P. Williams, The DixmierDouady classes of certain groupoid $C^{*}$-algebras with continuous trace, J. Operator Theory 81 (2019), 407-431.

[IW12] Marius Ionescu and Dana P. Williams, Remarks on the ideal structure of Fell bundle $C^{*}$-algebras, Houston J. Math. 38 (2012), 1241-1260.

[Kle65] Adam Kleppner, Multipliers of abelian groups, Math. Ann. 158 (1965), 11-34.

[Kum83] Alexander Kumjian, Diagonals in algebras of continuous trace, Operator algebras and their connections with topology and ergodic theory, Lecture Notes in Mathematics, vol. 1132, Springer-Verlag, Buşteni, Romania, 1983, pp. 297-311.

[Kum86] _ On $C^{*}$-diagonals, Canad. J. Math. 38 (1986), 969-1008.

[Kum88] _ On equivariant sheaf cohomology and elementary $C^{*}$-bundles, J. Operator Theory 20 (1988), 207-240.

[Mac58] George W. Mackey, Unitary representations of group extensions. I, Acta math. 99 (1958), 265-311.

[MRW96] Paul S. Muhly, Jean N. Renault, and Dana P. Williams, Continuous-trace groupoid $C^{*}$-algebras. III, Trans. Amer. Math. Soc. 348 (1996), 3621-3641.

[MW08] Paul S. Muhly and Dana P. Williams, Equivalence and disintegration theorems for Fell bundles and their $C^{*}$-algebras, Dissertationes Math. (Rozprawy Mat.) 456 (2008), 157.

[MW92] _ Continuous trace groupoid $C^{*}$-algebras. II, Math. Scand. 70 (1992), 127-145.

[MW95] _ Groupoid cohomology and the Dixmier-Douady class, Proc. London Math. Soc. (3) (1995), 109-134.

[Ren08] Jean N. Renault, Cartan subalgebras in $C^{*}$-algebras, Irish Math. Soc. Bull. 61 (2008), $29-63$.

[Ren15] — Topological amenability is a Borel property, Math. Scand. 117 (2015), 5-30.

[Ren87] _ Représentation des produits croisés d'algèbres de groupoïdes, J. Operator Theory 18 (1987), 67-97.

[SW13] Aidan Sims and Dana P. Williams, Amenability for Fell bundles over groupoids, Illinois J. Math. 57 (2013), 429-444.

[Tu06] Jean-Louis Tu, Groupoid cohomology and extensions, Trans. Amer. Math. Soc. 358 (2006), 4721-4747.

[Wil07] Dana P. Williams, Crossed products of $C^{*}$-algebras, Mathematical Surveys and Monographs, vol. 134, American Mathematical Society, Providence, RI, 2007.

[Wil19] _ A tool kit for groupoid $C^{*}$-algebras, Mathematical Surveys and Monographs, vol. 241, American Mathematical Society, Providence, RI, 2019. 
Department of Mathematics

United States Naval Academy

Annapolis, MD 21402 USA

ionescu@usna.edu

Department of Mathematics

University of Nevada

Reno NV 89557 USA

alex@unr.edu

Institut Denis Poisson (UMR 7013)

Université d'Orléans et CNRS

45067 Orléans Cedex 2, France

jean.renault@univ-orleans.fr

School of Mathematics and Applied Statistics

University of Wollongong

NSW 2522, Australia

asims@uow.edu.au

Department of Mathematics

Dartmouth College

Hanover, NH 03755-3551 USA

dana.williams@Dartmouth.edu 